University of Vermont

UVM ScholarWorks

2015

\title{
Acoustic streaming, fluid mixing, and particle transport by a Gaussian ultrasound beam in a cylindrical container
}

Jeffrey S. Marshall

University of Vermont

Junru Wu

University of Vermont

Follow this and additional works at: https://scholarworks.uvm.edu/cemsfac

Part of the Climate Commons

\section{Recommended Citation}

Marshall JS, Wu J. Acoustic streaming, fluid mixing, and particle transport by a Gaussian ultrasound beam in a cylindrical container. Physics of Fluids. 2015 Oct 7;27(10):103601.

This Article is brought to you for free and open access by the College of Engineering and Mathematical Sciences at UVM ScholarWorks. It has been accepted for inclusion in College of Engineering and Mathematical Sciences Faculty Publications by an authorized administrator of UVM ScholarWorks. For more information, please contact scholarworks@uvm.edu. 


\section{Acoustic streaming, fluid mixing, and particle transport by a Gaussian ultrasound beam in a cylindrical container}

Cite as: Phys. Fluids 27, 103601 (2015); https://doi.org/10.1063/1.4932232

Submitted: 18 June 2015 . Accepted: 21 September 2015 . Published Online: 07 October 2015

Jeffrey S. Marshall, and Junru Wu
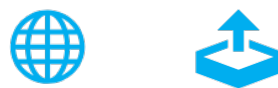

View Online

\section{ARTICLES YOU MAY BE INTERESTED IN}

Acoustic streaming and thermal instability of flow generated by ultrasound in a cylindrical container

Physics of Fluids 28, 104105 (2016); https://doi.org/10.1063/1.4965899

\section{Acoustic Streaming near a Boundary}

The Journal of the Acoustical Society of America 30, 329 (1958); https://

doi.org/10.1121/1.1909587

Scaling and dimensional analysis of acoustic streaming jets

Physics of Fluids 26, 093602 (2014); https://doi.org/10.1063/1.4895518

\section{Physics of Fluids GALLERY OF COVERS}




\title{
Acoustic streaming, fluid mixing, and particle transport by a Gaussian ultrasound beam in a cylindrical container
}

\author{
Jeffrey S. Marshall ${ }^{1, a)}$ and Junru $\mathrm{Wu}^{2}$ \\ ${ }^{1}$ School of Engineering, The University of Vermont, Burlington, Vermont 05405, USA \\ ${ }^{2}$ Department of Physics, The University of Vermont, Burlington, Vermont 05405, USA
}

(Received 18 June 2015; accepted 21 September 2015; published online 7 October 2015)

\begin{abstract}
A computational study is reported of the acoustic streaming flow field generated by a Gaussian ultrasound beam propagating normally toward the end wall of a cylindrical container. Particular focus is given to examining the effectiveness of the acoustic streaming flow for fluid mixing within the container, for deposition of particles in suspension onto the bottom surface, and for particle suspension from the bottom surface back into the flow field. The flow field is assumed to be axisymmetric with the ultrasound transducer oriented parallel to the cylinder axis and normal to the bottom surface of the container, which we refer to as the impingement surface. Reflection of the sound from the impingement surface and sound absorption within the material at the container bottom are both accounted for in the computation. The computation also accounts for thermal buoyancy force due to ultrasonic heating of the impingement surface, but over the time period considered in the current simulations, the flow is found to be dominated by the acoustic streaming force, with only moderate effect of buoyancy force. (C) 2015 AIP Publishing LLC. [http://dx.doi.org/10.1063/1.4932232]
\end{abstract}

\section{INTRODUCTION}

Application of ultrasound in a container, where the ultrasound propagates downward toward the bottom surface of the container, generates an acoustic streaming flow that leads to circulatory fluid motion within the container. Ultrasound is used in a wide variety of fluid processes, both to mix ingredients and to break up heterogeneities in the fluid. Some common applications include blending of multiphase compounds (such as cement), ${ }^{1,2}$ mixing of biological assays, ${ }^{3}$ mixing of fluids within microfluidic devices, ${ }^{4,5}$ mixing of food products such as bread dough or chocolate, ${ }^{6}$ mixing of resins and curing agents during manufacture of plastics and composites, ${ }^{7}$ mixing of antimicrobial fluids in dental operations, ${ }^{8}$ and enhanced blending of chemical compounds during chemical reactions. ${ }^{9-11}$ Numerous companies now sell ultrasonic mixers and applications are rapidly expanding.

Ultrasonic cleaners are used for removal of particles from a surface, particularly in the wafer manufacturing industry. Use of focused ultrasound directed toward a surface in a liquid bath can produce small cavitation bubbles on the surface. ${ }^{12-14}$ Lamminen et al. ${ }^{15}$ showed that oscillation and collapse of the cavitation bubbles act to detach particles from the surface and acoustic streaming associated with oscillating bubbles acts to transport particles away from the surface. Ultrasonic cleaning efficiency is improved by use of an ionic solution, for which electrostatic field forces contribute to removal of the particles from the surface. ${ }^{16}$ Effectiveness of acoustic forcing for surface cleaning in air was demonstrated by Chen and $\mathrm{Wu} .{ }^{17}$ Fuhrmann et al. ${ }^{18}$ showed that a standing acoustic wave can effectively improve the efficiency of an aerodynamic particle removal approach.

Acoustic streaming and radiation forces have also been used successfully to manipulate particles on a surface and to sort particles by size. ${ }^{19,20} \mathrm{~A}$ few applications have used ultrasound to

\footnotetext{
a) Author to whom correspondence should be addressed. Electronic mail: jeffm@cems.uvm.edu. Telephone: 1 (802) 6563826.
} 
deposit adhesive particles held in suspension onto a surface. For instance, a common practice in ultrasound-assisted drug delivery is to confine the drug within a liposome, which is then transported to the target site via an ultrasonic wave. ${ }^{21}$ Ultrasound can also be used in such applications to control release of the drug from the liposomes. ${ }^{22} \mathrm{Ma}$ et al..$^{23}$ recently reported successful development of a system that uses ultrasound to transport liposomes filled with antimicrobial fluid from a suspension in a microplate well to a biofilm along the well bottom, and which then further uses ultrasound to enhance liposome penetration into the biofilm. The method was shown to successfully kill bacterial cells within the biofilm when the antimicrobial fluid was released following liposome bursting.

Acoustic waves have also been used to induce particle clustering at the nodal points of the sound waves. Wiklund et $a .^{24}$ reported on an ultrasonic particle concentrator that uses a standing-wave resonator to induce clustering of particles from suspension in a 96-well microplate array. Agrawal et al..$^{25}$ showed that significant particle transport and clustering can be obtained by acoustic streaming even at very low frequency oscillations (e.g., $100 \mathrm{~Hz}$ ) using an experiment in which particles collect at the nodes of a capillary wave. A theoretical and numerical study explaining the physics of particle clustering in oscillatory straining flows was given by Marshall. ${ }^{26}$

A series of simulations of acoustic streaming motions in a two-dimensional rectangular container were reported by Aktas and Farouk, ${ }^{27}$ where the streaming was produced by oscillation of the left-hand wall of the rectangular container. The simulations exhibited different numbers of circulatory cells in the container depending on the container aspect ratio and the amplitude of wall motion. Extension of this work for differentially heated top and bottom walls was reported by Aktas and Ozgumus. ${ }^{28}$ Nonlinear acoustic streaming in a two-dimensional rectangular container was investigated computationally by Daru et al. ${ }^{29}$ An experimental study examining the effect of two different bottom materials on acoustic streaming in a closed cylindrical container was reported by Wiklund et al.,$^{30}$ which used particle image velocimetry (PIV) to measure the ring-like vortical acoustic streaming flow in the container. Suri et $a l .{ }^{31}$ showed that off-axis application of ultrasound in a cylindrical container can be used to induce chaotic mixing. In these experiments, two ultrasound transducers were used, one aligned axially at some offset distance from the cylinder axis and one placed at the side of the cylinder. An experimental study of the acoustic streaming transient flow field was reported by Mitome et al. ${ }^{32}$ for streaming flow in a large rectangular container, activated by a transducer placed at the container side. Because the transducer was much smaller than the dimensions of this container, the container wall boundaries did not play a significant role in the transient flow development.

Several recent studies have examined thermal effects in acoustic streaming flows, driven in part by the fact that ultrasound emissions also lead to heating of the container walls through absorption of the sound energy. The effect of a horizontal temperature gradient in a cavity subject to horizontal acoustic wave propagation was examined numerically by Dridi et al. ${ }^{33,34}$ The flow field discussed in these papers exhibited a series of bifurcations as the temperature gradient and acoustic intensity were varied, where, in general, acoustic streaming was found to stabilize the thermal instability when the sound intensity is sufficiently strong. The effect of acoustic streaming on the RayleighBénard thermal instability problem, in which a vertical temperature gradient is applied on the fluid in a direction orthogonal to an acoustic streaming flow along a channel, induced by horizontal acoustic emissions, was examined numerically by Hadid et al. ${ }^{35}$ A numerical study of thermoviscous effects, arising mainly through the temperature and density dependence of the fluid viscosity, on ultrasound-induced acoustic streaming in microchannels was reported by Muller and Bruus. ${ }^{36}$

The current paper reports on a computational study of acoustic streaming flow generated by a Gaussian ultrasound beam propagating along the axis of a cylindrical container, directed toward the container end-wall. The primary objective of the study was to characterize the potential of the flow for fluid mixing, particle lift-off from the bottom surface, and deposition of adhesive particles onto the bottom surface with different container heights and end-wall acoustic reflection coefficients. The flow simulations were performed using an axisymmetric vorticity-streamfunction method. Thermal heating of the end-wall material is computed and thermal buoyancy effects on the fluid field are included in the flow computation, although for the time period and parameter values used in the simulations thermal buoyancy is of secondary importance to the acoustic streaming force. 
The governing equations for the flow and temperature field and the computational method are presented in Section II. A scaling analysis is also presented in this section to extract velocity and temperature scales for this problem, which are subsequently used to define dimensionless variables. Results for the flow and temperature fields are presented in Section III, followed by a discussion of the effectiveness of this flow field for fluid mixing, particle removal from the surface, and particle deposition onto the surface. Conclusions are presented in Section IV.

\section{GOVERNING EQUATIONS AND COMPUTATIONAL METHOD FOR ACOUSTIC STREAMING}

The flow field is generated by an ultrasound transducer oriented along the axis of a cylindrical container of radius $L$. The transducer is located along the top surface of a liquid of depth $H$ and is oriented normal to a solid end-wall of the container, which is a solid with thickness $D$. A Gaussian acoustic beam of radius $b$ propagates downward toward the cylinder end wall. The problem is analyzed using a cylindrical polar coordinate system $(r, \theta, z)$ as shown in Figure 1. The velocity, acoustic intensity, and temperature fields are assumed to be axisymmetric.

\section{A. Governing equations for fluid and thermal fields}

The fluid velocity field consists of an oscillating (ac) flow $\mathbf{u}_{a c}$ generated by the acoustic wave, a second-order acoustic streaming (dc) flow generated by second-order effects, and a non-oscillatory flow generated by thermal buoyancy. The second and third components of the velocity field are lumped into the dc field $\mathbf{u}$, which varies in time but over a much longer time span than the acoustic period $\tau=1 / f$, where $f$ is the acoustic frequency. Using the Boussinesq approximation with low fluid Mach number, the governing equations for the dc velocity field are given by the continuity and Navier-Stokes equations as

$$
\nabla \cdot \mathbf{u}=0, \quad \frac{\partial \mathbf{u}}{\partial t}+(\mathbf{u} \cdot \nabla) \mathbf{u}=-\frac{1}{\rho_{0 F}} \nabla p+g \beta\left(T-T_{A}\right) \mathbf{e}_{z}+v \nabla^{2} \mathbf{u}+\frac{1}{\rho_{0 F}} \mathbf{F} .
$$

In these equations, $\rho_{0 F}$ is the average fluid density, $g$ is gravitational acceleration, $\beta$ is the coefficient of thermal expansion, $v$ is the kinematic viscosity, $T$ is temperature, and $T_{A}$ is the ambient temperature. The acoustic forcing term $\mathbf{F}$ is given by $\mathrm{Nyborg}^{37}$ as

$$
\mathbf{F}=-\rho_{0 F}\left\langle\left(\mathbf{u}_{a c} \cdot \nabla\right) \mathbf{u}_{a c}+\mathbf{u}_{a c}\left(\nabla \cdot \mathbf{u}_{a c}\right)\right\rangle,
$$

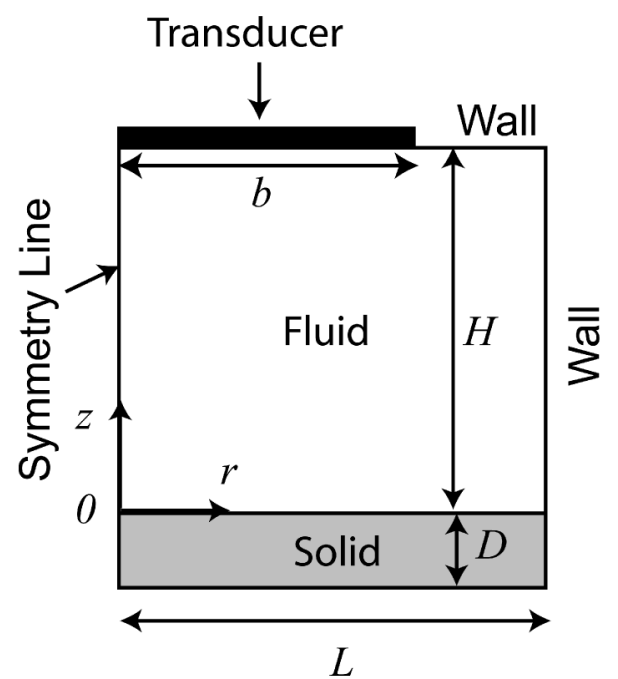

FIG. 1. Sketch showing the computational domain used for the computations, showing the $r-z$ plane of a cylindrical polar coordinate system. 
where $\langle\cdot\rangle$ denotes an average over the acoustic period $\tau$. The first-order ac velocity field and the ac acoustic pressure field satisfy

$$
\rho_{0 F} \frac{\partial \mathbf{u}_{a c}}{\partial t}=-\nabla p_{a c}, \quad \frac{\partial^{2} p_{a c}}{\partial t^{2}}=c_{F}^{2} \nabla^{2} p_{a c},
$$

where $c_{F}$ is the sound speed in the fluid. We let the radius of the beam source transducer $a$ satisfy the condition that $k_{F} a>>1$, where $k_{F}$ is the acoustic wave number in the fluid, and employ the parabolic approximation ${ }^{38,39}$ to write the acoustic body force $\mathbf{F}$ as ${ }^{37,40-42}$

$$
\mathbf{F}=\frac{2 \alpha_{F}}{c_{F}} \mathbf{I}
$$

where $\alpha_{F}$ is the acoustic pressure attenuation coefficient within the fluid.

The temperature field within the fluid is governed by the heat equation

$$
\frac{\partial T}{\partial t}+\mathbf{u} \cdot \nabla T=\eta_{F} \nabla^{2} T+\frac{q_{F}}{\rho_{0 F} \lambda_{F}},
$$

where $\eta_{F}=\gamma_{F} / \rho_{0 F} \lambda_{F}$ is the thermal diffusivity in the fluid, $\gamma_{F}$ is the fluid thermal conductivity, $q_{F}$ is the fluid volumetric heat supply, and $\lambda_{F}$ is the fluid specific heat. In the solid, the temperature is governed by

$$
\frac{\partial T}{\partial t}=\eta_{S} \nabla^{2} T+\frac{q_{S}}{\rho_{0 S} \lambda_{S}},
$$

where $\eta_{S}=\gamma_{S} / \rho_{0 S} \lambda_{S}$ is the thermal diffusivity in the solid, $\gamma_{S}$ is the solid thermal conductivity, $q_{S}$ is the solid volumetric heat supply, and $\lambda_{S}$ is the solid specific heat. The volumetric heat supply terms associated with attenuation of the acoustic beam in the fluid and solid, respectively, are given by ${ }^{43}$

$$
q_{F}=2 \alpha_{F} I, \quad q_{S}=2 \alpha_{S} I,
$$

where $I=|\mathbf{I}|$ is the acoustic intensity magnitude.

\section{B. Acoustic intensity field}

In order to simplify the analysis, the acoustic wave generated by the transducer is assumed to have the form of a Gaussian beam. This simplification is supported by the analysis and experimental results of Moudjed et al. ${ }^{44}$ who showed that the acoustic streaming generated by an acoustic transducer is closely approximated by that generated by a simple parallel wave approximation, even in the transducer near field.

An analysis is given here for the case where the incident beam is normal to the bottom surface; the more general case of arbitrary beam angle is examined by Norris. ${ }^{45}$ The acoustic pressure of a Gaussian beam in the fluid generated by the transducer, $p_{i}$, and the reflected beam, $p_{r}$, from the fluid-solid interface at $z=0$ can be written as ${ }^{46,47}$

$$
\begin{gathered}
p_{i}(r, z)=p_{0}\left(A_{1}(z) / B\right)^{1 / 2} \exp \left[-A_{1}(z)(r / b)^{2}\right] \cos \left[k_{F}(H-z)-\sigma t\right] \exp \left[-\alpha_{F}(H-z)\right], \\
p_{r}(r, z)=R p_{0}\left(A_{2}(z) / B\right)^{1 / 2} \exp \left[-A_{2}(z)(r / b)^{2}\right] \cos \left(k_{F} z-\sigma t\right) \exp \left[-\alpha_{F} z\right],
\end{gathered}
$$

where $p_{0}$ is the acoustic pressure amplitude at the transducer surface, $R$ is the acoustic pressure reflection coefficient, $b$ is the beam width, $f$ is the acoustic frequency with circular frequency $\sigma=2 \pi f$, and the diffraction coefficients $A_{1}$ and $A_{2}$ are given by

$$
A_{1}(z)=\frac{B}{1+B^{2}\left(\frac{H-z}{r_{0 F}}\right)^{2}}, \quad A_{2}(z)=\frac{A_{1}(0)}{1+A_{1}^{2}(0)\left(z / r_{0 F}\right)^{2}} .
$$

In (9), $B$ is the Gaussian coefficient at the transducer, $r_{0 F}=\pi b^{2} f / c_{F}$ is the Rayleigh distance in the fluid, and $H-z$ and $z$ are the distances to the top of the fluid container and to the fluid-solid interface, respectively, from a point with coordinates $(r, z)$ within the fluid domain. The temporally 
averaged acoustic intensity with reflection can be expressed by

$$
I(r, z)=f \int_{0}^{1 / f} p_{t} v_{t} d t=f\left(\frac{p_{0}^{2}}{\rho_{0 F} c_{F}}\right) \int_{0}^{1 / f}\left(p_{i}+p_{r}\right)\left(p_{i}-p_{r}\right) d t,
$$

where the total acoustic pressure is $p_{t}=p_{i}+p_{r}$ and the particle velocity is $v_{t}=v_{i}+v_{r}=\frac{p_{i}}{\rho_{0 F} c_{F}}+$ $\frac{p_{r}}{\rho_{0 F}^{\left(-c_{F}\right)}}$. Substituting (8) into (10) and performing the integration gives the acoustic intensity in the fluid as

$$
\begin{aligned}
I(r, z) & =I_{0}\left[A_{1}(z) / B\right] \exp \left[-2 A_{1}(z)(r / b)^{2}\right] \exp \left[-2 \alpha_{F}(H-z)\right] \\
& -R^{2} I_{0}\left[A_{2}(z) / B\right] \exp \left[-2 A_{2}(z)(r / b)^{2}\right] \exp \left[-2 \alpha_{F} z\right],
\end{aligned}
$$

where $I_{0}$ is the centerline acoustic intensity at the transducer surface.

The acoustic intensity of the Gaussian beam that penetrates into the solid $(z<0)$ is given by

$$
I(r, z)=\left(1-R^{2}\right) I_{0}\left[A_{3}(z) / B\right] \exp \left[-2 A_{3}(z)(r / b)^{2}\right] \exp \left(2 \alpha_{S} z\right) .
$$

The diffraction function $A_{3}(z)$ has the same form as $A_{2}(z)$ in (9), but with $r_{0 F}$ replaced by the Rayleigh distance $r_{0 S}=\pi b^{2} f / c_{S}$ in the solid. While for completeness we have shown the attenuation terms in the fluid in (11), as well as the acoustic heating term in the fluid in (5), it is noted that in many cases these terms have very small effect since the acoustic attenuation coefficient in the fluid (e.g., water, $\alpha_{F} \cong 0.056 \mathrm{~m}^{-1}$ ) is often small compared to that in the solid (e.g., polyurethane, $\left.\alpha_{S} \cong 140 \mathrm{~m}^{-1}\right)$, where values above are given for ultrasound frequency $f=2.25 \mathrm{MHz} .{ }^{47}$

\section{Scaling and dimensionless equations}

The variables are non-dimensionalized using the initial beam width $b$ as a length scale and the convective time scale $t_{0}=b / U_{0}$. Since the cylindrical vessel is sufficiently short that the entire domain can be considered to lie in the near-field of the acoustic streaming jet, a velocity scale $U_{0}$ can be determined by balancing the order of magnitude of the inertia and acoustic forcing terms, ${ }^{42}$ giving

$$
U_{0}=\left(\frac{2 \alpha_{F} b}{\rho_{0 F} c_{F}} I_{0}\right)^{1 / 2}
$$

The scale of the temperature increase $T_{0}$ is determined by balancing the acoustic energy flux $I_{0}$ on the solid surface with the convective energy loss flux from the surface, given by Newton's law of cooling as $h\left(T-T_{A}\right)$ where $h$ is the heat transfer coefficient at the liquid-solid interface, giving the scale of the temperature change as

$$
T_{0}=\frac{I_{0}}{h} .
$$

Using these scales, dimensionless variables are defined (using primes) by

$$
\begin{array}{llll}
\mathbf{u}^{\prime}=\mathbf{u} / U_{0}, & T^{\prime}=\left(T-T_{A}\right) / T_{0}, & \boldsymbol{\omega}^{\prime}=\boldsymbol{\omega} t_{0}, & \psi^{\prime}=\psi / U_{0} b^{2} \\
I^{\prime}=I / I_{0}, & \mathbf{x}^{\prime}=\mathbf{x} / b, & t^{\prime}=t / t_{0} . &
\end{array}
$$

In the above, $\boldsymbol{\omega}=\nabla \times \mathbf{u}$ is the vorticity vector and $\psi$ is the Stokes streamfunction, defined for axisymmetric flows in terms of the velocity components $\mathbf{u}=u(r, z, t) \mathbf{e}_{r}+w(r, z, t) \mathbf{e}_{z}$ by

$$
u=-\frac{1}{r} \frac{\partial \psi}{\partial z}, \quad w=\frac{1}{r} \frac{\partial \psi}{\partial r} .
$$

Dropping the primes on the dimensionless variables, the governing equations for axisymmetric flow are written in terms of the vorticity $\boldsymbol{\omega}=\omega(r, z, t) \mathbf{e}_{\theta}$ and the Stokes stream function as

$$
\frac{\partial^{2} \psi}{\partial r^{2}}-\frac{1}{r} \frac{\partial \psi}{\partial r}+\frac{\partial^{2} \psi}{\partial z^{2}}=-r \omega
$$




$$
\frac{\partial \omega}{\partial t}+u \frac{\partial \omega}{\partial r}-\frac{u \omega}{r}+w \frac{\partial \omega}{\partial z}=-\frac{\mathrm{Gr}}{\operatorname{Re}^{2}} \frac{\partial T}{\partial r}+\frac{\partial I}{\partial r}+\frac{1}{\operatorname{Re}}\left[\frac{\partial^{2} \omega}{\partial r^{2}}+\frac{1}{r} \frac{\partial \omega}{\partial r}-\frac{\omega}{r^{2}}+\frac{\partial^{2} \omega}{\partial z^{2}}\right]
$$

where $\operatorname{Re}=U_{0} b / v$ and $\mathrm{Gr}=g \beta T_{0} b^{3} / v^{2}$ are the Reynolds and Grashof numbers. The governing equations for the dimensionless temperature field in the fluid and in the solid are

$$
\begin{gathered}
\frac{\partial T}{\partial t}+u \frac{\partial T}{\partial r}+w \frac{\partial T}{\partial z}=\frac{1}{\operatorname{Pr}_{F} \operatorname{Re}}\left[\frac{\partial^{2} T}{\partial r^{2}}+\frac{1}{r} \frac{\partial T}{\partial r}+\frac{\partial^{2} T}{\partial z^{2}}\right]+\frac{2 \alpha_{F}^{\prime} I_{0}}{\rho_{0 F} \lambda_{F} T_{0} U_{0}} I(r, z) \text { for } z>0, \\
\frac{\partial T}{\partial t}=\frac{1}{\operatorname{Pr}_{S} \operatorname{Re}}\left[\frac{\partial^{2} T}{\partial r^{2}}+\frac{1}{r} \frac{\partial T}{\partial r}+\frac{\partial^{2} T}{\partial z^{2}}\right]+\frac{2 \alpha_{S}^{\prime} I_{0}}{\rho_{0 S} \lambda_{S} T_{0} U_{0}} I(r, z) \text { for } z \leq 0,
\end{gathered}
$$

where $\operatorname{Pr}_{F}=v / \eta_{F}$ and $\operatorname{Pr}_{S}=v / \eta_{S}$ are the Prandtl numbers in the fluid and solid, respectively, and $\alpha_{F}^{\prime}=\alpha_{F} b$ and $\alpha_{S}^{\prime}=\alpha_{S} b$ are the corresponding dimensionless attenuation coefficients.

The solution domain consists of a cylindrical container with no-slip walls on the side, top, and bottom. Acoustic streaming leads to a non-zero effective slip velocity on the walls given by Rayleigh as

$$
u_{\text {slip }}=-\frac{3}{4} \sigma^{-1} U(x) \frac{d U}{d x}
$$

where $U(x)$ is the inviscid velocity just outside the viscous boundary layer and $x$ is distance along the boundary in the direction of flow. It is assumed that the sound frequency is in the ultrasonic range, so that $\sigma^{-1}$ is sufficiently small that the Rayleigh slip may be neglected (i.e., $\sigma b / U_{0}>>1$ ). With this approximation, the dimensionless boundary conditions on the sides of the computational domain are given by

$$
\begin{array}{llll}
\text { on } r=0: & u=0, & \omega=-\frac{\partial w}{\partial r}=0, & \frac{\partial T}{\partial r}=0, \\
\text { on } r=L / b: & u=0, & w=0, & \frac{\gamma_{w}}{\gamma_{F}} \frac{b}{\tau_{w}}\left(T-T_{\text {ext }}\right)+\frac{\partial T}{\partial r}=0, \\
\text { on } z=H / b: & u=0, & w=0, & \frac{\partial T}{\partial z}=0, \\
\text { on } z=-D / b: & u=0, & w=0, & \frac{\partial T}{\partial z}=0 .
\end{array}
$$

In this equation, $\gamma_{w}$ is the thermal conductivity of the side wall, $\tau_{w}$ is the side wall thickness, and $T_{\text {ext }}$ is a constant temperature external to the side wall (which is set equal to the initial temperature within the computational domain). At the interface $z=0$ between the solid and the fluid, the boundary condition is

$$
u=0, \quad w=0,\left.\quad \gamma_{F} \frac{\partial T}{\partial z}\right|_{z=0^{+}}=\left.\gamma_{S} \frac{\partial T}{\partial z}\right|_{z=0^{-}},\left.\quad T\right|_{z=0^{+}}=\left.T\right|_{z=0^{-}}
$$

\section{Computational method}

Systems (17)-(20) are solved using the Crank-Nicholson time advancement algorithm. The spatial derivatives are approximated by centered differences in the diffusive terms and by upstreamweighted differences in the convective terms, where the latter are given by

$$
\left.u \frac{\partial T}{\partial r}\right|_{i, j} \cong \frac{1}{2 \Delta r} u_{i, j}\left[\left(1-\varepsilon_{i, j}\right)\left(T_{i+1, j}-T_{i, j}\right)+\left(1+\varepsilon_{i, j}\right)\left(T_{i, j}-T_{i-1, j}\right)\right]
$$

and where $\varepsilon_{i, j}=\gamma \operatorname{sign}\left(u_{i, j}\right)$. The coefficient $\gamma$ is a weighting constant, such that $0 \leq \gamma \leq 1$, where $\gamma=0$ corresponds to the second-order centered difference scheme and $\gamma=1$ corresponds to the first-order upstream difference. It was found that robust results with no sign of convective 
TABLE I. Dimensionless parameters used for the "basic problem" in the numerical simulations, with assumed ultrasound frequency $2.25 \mathrm{MHz}$.

\begin{tabular}{lclc}
\hline \multicolumn{1}{c}{ Parameters for fluid and container } & & \multicolumn{1}{c}{ Parameters for end-wall solid (polyurethane) } \\
\hline Reynolds number, Re & 400 & Solid Prandtl number, $\operatorname{Pr}_{S}$ & 2.2 \\
Grashof number, Gr & 300 & Acoustic reflection coefficient, $R$ & 0.08 \\
Fluid Prandtl number, $\operatorname{Pr}_{F}$ & 4.6 & Attenuation coefficient, $\alpha_{S} b$ & 1.5 \\
Volumetric thermal expansion coefficient, $\beta T_{0}$ & 0.0004 & Solid Rayleigh distance, $r_{0} / b$ & 47 \\
Fluid height, $H / b$ & 1.0 & Thermal conductivity ratio, $\gamma_{F} / \gamma_{S}$ & 85 \\
Attenuation coefficient, $\alpha_{F} b$ & 0.0006 & & \\
Container radius, $L / b$ & 1.5 & & \\
Solid thickness, $D / b$ & 0.1 & & \\
Fluid Rayleigh distance, $r_{0} / b$ & 55 & & \\
Duty cycle & $10 \%$ & & \\
Acoustic wavelength, $\lambda / b$ & 0.063 & & \\
\hline
\end{tabular}

instability were obtained with $10 \%$ upstream differencing for the vorticity transport equation and $30 \%$ upstream difference for the heat and scalar concentration transport equations.

The boundary conditions for the streamfunction are chosen to satisfy the no-penetration condition on the boundaries, and the boundary conditions for vorticity are chosen to satisfy the symmetry condition on $r=0$ and the no-slip condition on the other boundaries. The vorticity boundary condition on a no-slip wall is specific using the Thom equation. ${ }^{48}$ The equation for the axial velocity on the symmetry axis can be reduced using a Taylor series expansion about $r=0$ to

$$
w(0, z, t)=\left.2 \frac{\partial^{2} \psi}{\partial r^{2}}\right|_{r=0} .
$$

A Gauss-Seidel iteration method is used to solve the resulting matrix equation with a relative error of $10^{-6}$ and dimensionless time step of $\Delta t=0.001$. The Courant-Friedrichs-Lewy (CFL) number is less than 0.1 for all computations.

The flow problem has a large number of dimensionless parameters. Many of these parameters were held constant in the paper with values corresponding to a "basic problem," and then values were varied only for a selected group of parameters that most influenced the problem under investigation. The basic problem specification was motivated by applications in which ultrasound is used for mixing or particle manipulation, such as in a microplate well, as is used in several biological sampling applications. Solid properties were based on polyurethane end wall material, which has a low acoustic reflection coefficient. ${ }^{49}$ Detailed parameter values are given in Table I.

A grid independence study was performed with four different grids, labeled grid A-D, ranging between about 30000 and over $2.2 \times 10^{6}$ evenly spaced grid points in the $r$ - $z$ plane. Maximum and minimum values of different parameters in the steady-state condition are listed in Table II. The flow fields on all four grids were similar, although for the most refined grid (grid A) the grid cells were sufficiently small that computer round-off error started to degrade the solution, particularly for the streamfunction equation. The computations reported in the paper were conducted using grid $\mathrm{B}$, or a grid with the same resolution as grid B for cases with variable container height.

TABLE II. Steady-state values for grids A-D, with $N$ grid points.

\begin{tabular}{lrccccc}
\hline \hline Grid & \multicolumn{1}{c}{$N$} & $T_{\max }$ & $T_{s, \max }$ & $\omega_{\max }$ & $\omega_{\min }$ & $w_{\min }$ \\
\hline A & 2203201 & 0.5739 & 0.01201 & 2.880 & -1.313 & -0.1094 \\
B & 551601 & 0.5701 & 0.01234 & 2.961 & -1.199 & -0.1120 \\
C & 125751 & 0.6786 & 0.01339 & 2.928 & -1.185 & -0.1106 \\
D & 31626 & 0.6664 & 0.01320 & 2.917 & -1.181 & -0.1104 \\
\hline \hline
\end{tabular}




\section{ACOUSTIC STREAMING FIELD}

\section{A. Flow and temperature fields}

The acoustic field is activated at time $t=0$, at which time the fluid is at rest and both the fluid and its container are at the ambient temperature $T_{A}$. The ultrasound beam has two significant effects-(a) the acoustic intensity gives rise to a body force within the fluid, given by (4), which induces a downward flow oriented toward the cylinder end-wall and (b) the sound absorption within the end-wall solid heats up the end-wall material. Both of these effects occur fairly quickly after the impulsive start and evolve toward a quasi-steady state flow field. For instance, Figure 2 shows profiles of the fluid axial velocity $w$ and solid temperature $T$ at different times after the impulsive start, leading up to the steady state solutions (indicated by dashed lines). The downward axial velocity field (Figure 2(a)) is driven by a region of negative azimuthal vorticity which is generated by the radial gradient of the acoustic body force. The resulting downward axial flow vanishes at both the transducer surface and the impingement surface (the cylinder end-wall), and it attains a maximum at a height of about $40 \%$ of the distance from the end-wall surface to the transducer. We note that all variables plotted here and elsewhere in the paper refer to time-averages over the period of the ultrasound wave. The temperature field of the solid, shown in Figure 2(b), has a maximum value on the bottom surface of the end-wall material, at which an adiabatic boundary condition is imposed. The heat flux into the fluid leads to a slightly increased top surface temperature of the end-wall material, but the change in top surface temperature is nearly two orders of magnitude smaller than the maximum bulk temperature change within the end-wall material.

Contour plots of the steady-state flow field are giving in Figure 3, as well as streamlines of the acoustic streaming velocity field. The acoustic intensity (Fig. 3(a)) is observed to decrease rapidly within the solid as the sound is absorbed by the solid material, resulting in high temperature values within the most active absorption region in Fig. 3(b). The acoustic streaming velocity field (Fig. 3(c)) has the form of a confined vortex ring, with downward flow near the axis of the cylindrical container and upward return flow along the container sides. This acoustic streaming field is very similar in structure to the short-time quasi-steady state field reported in the experimental study of Ma et al. ${ }^{23}$ as well as to the acoustic streaming field shown in the review by Wiklund et al., ${ }^{30}$ both of which examine a similar configuration. The azimuthal vorticity field (Fig. 3(d)) is dominated by a large region of negative vorticity in a toroidal shape, which is generated by the acoustic body force, and thin regions of positive vorticity near the walls generated by the no-slip boundary condition.

\section{B. Effect of container height}

A series of computations were performed using the same parameter values as in the basic flow, but varying the container height from $H=1$ to 8 . Azimuthal vorticity contours and streamlines for

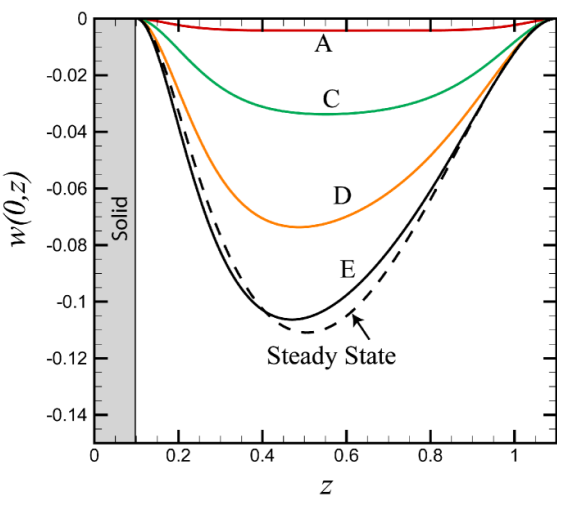

(a)

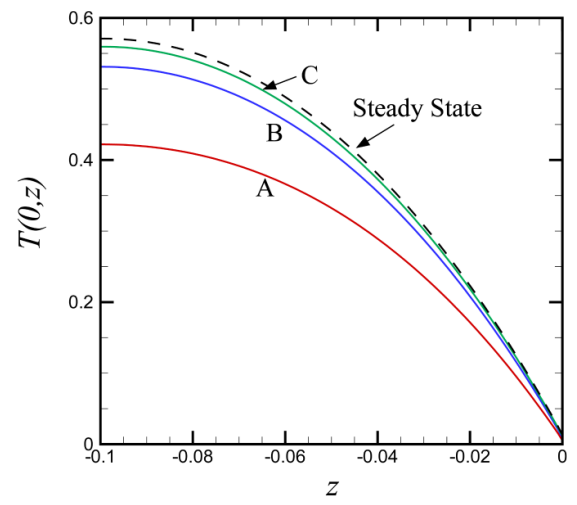

(b)

FIG. 2. Variation of (a) axial velocity and (b) temperature along the symmetry axis at times $t=5$ (A, red), 10 (B, blue), 15 (C, green line), 25 (D, orange line), 40 (E, black line), and at steady state (dashed line). Figure (b) is plotted only within the solid $(0 \leq z \leq 0.1)$, and in (a) the region occupied by the solid is indicated by grey shading. 


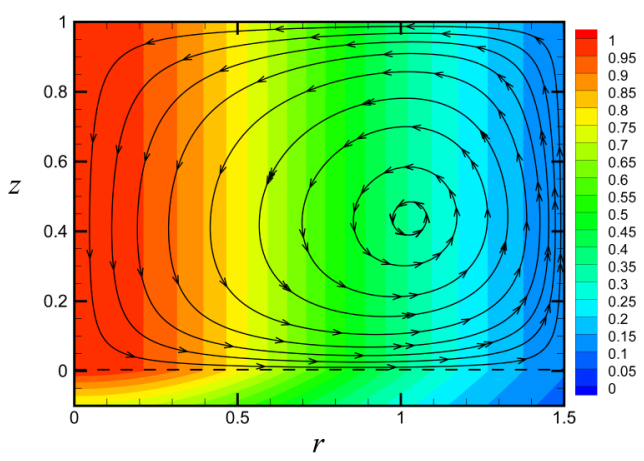

(a)

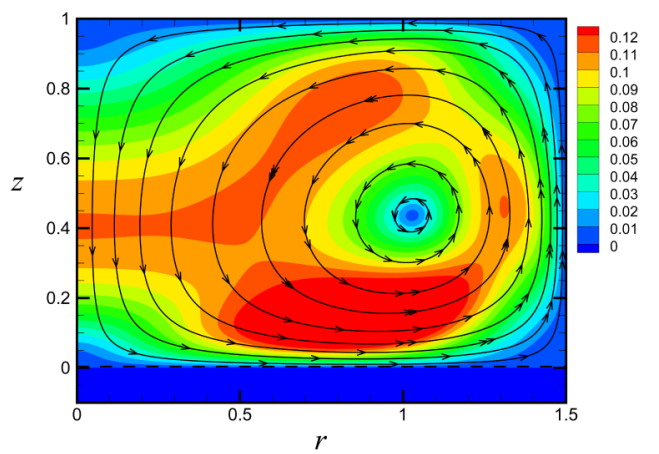

(c)

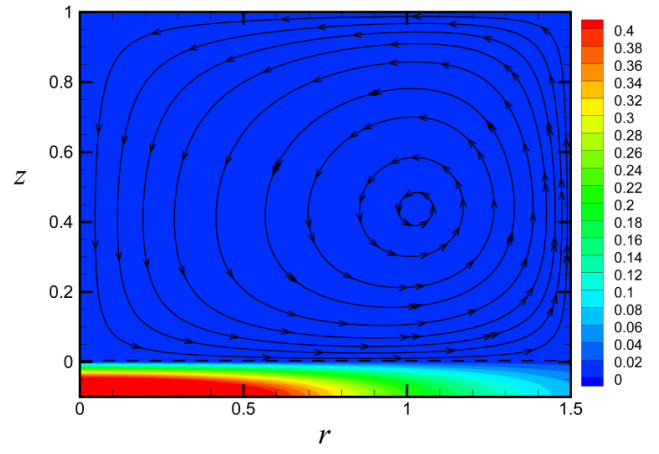

(b)

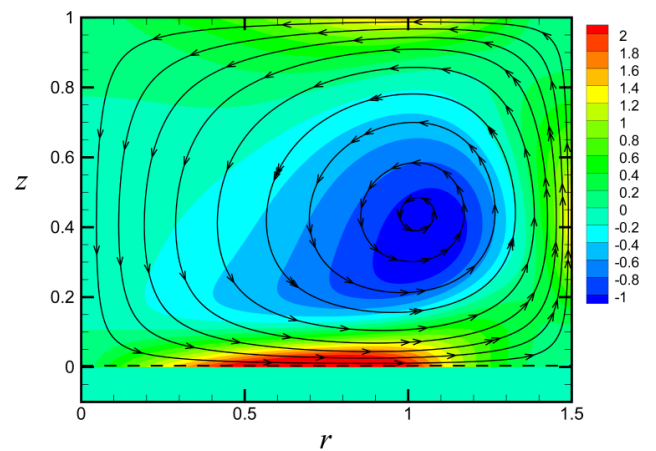

(d)

FIG. 3. Contour plots for dimensionless variables for the basic flow, including (a) magnitude of acoustic intensity, (b) temperature, (c) acoustic streaming velocity magnitude, and (d) azimuthal vorticity. The fluid is located above the dashed line at $z=0$ and the solid is located below this line. Streamlines indicate the direction of acoustic streaming flow in the quasi-equilibrium state.

cases with $H=2,4$, and 8 are shown in Figure 4, and the maximum and minimum values of azimuthal vorticity and the maximum value of the temperature are plotted as a function of $H$ in Figure 5. For all container heights examined, a single recirculating flow forms with center of circulation located between $z=0.5$ and 1 . The maximum temperature of the solid remains nearly constant as $\mathrm{H}$ changes, but a marked increase is observed in both $\omega_{\max }$ and $\left|\omega_{\min }\right|$ as $H$ increases, with nearly linear variation in each case. The fluid flow is driven by the acoustic streaming body force, which acts primarily when fluid elements are traveling within about a radius of unity from the cylinder axis, during the downward stroke of the recirculation pattern. This negative vorticity is pushed radially outward near the bottom of the fluid container, causing the vorticity to intensify as it stretches. Taller containers exhibit higher vorticity magnitudes than shorter containers because the fluid elements spend a longer time exposed to the acoustic body force as they travel downward near the fluid axis.

\section{Effect of reflection coefficient}

The effect of end-wall reflection coefficient $R$ on the flow field was evaluated by repeating the basic flow simulation with values of reflection coefficient $R=0.3$ and 0.6 , and all other parameters held fixed. The computations are performed with no acoustic reflection from the top surface, which is particularly reasonable either for low reflection coefficient values or for a transducer made of a polymeric material, such as Polyvinylidene fluoride (PVDF). Because of the lack of upper surface reflections and the low duty cycle, no standing wave effects are assumed to occur. The simulated flow field was observed to have the same qualitative structure for all values of $R$ examined. A plot showing the time variation of different quantities for cases with different reflection coefficients is given in Figure 6 , where the data are collapsed by dividing by the normalization factor $1-R^{2}$, which is the 


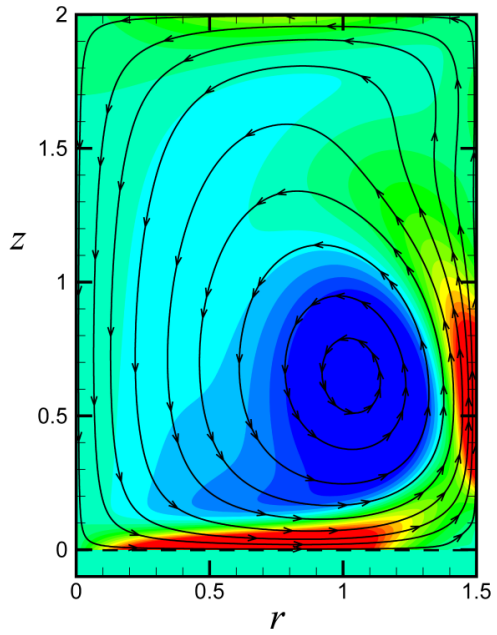

(a)

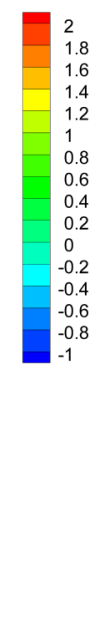

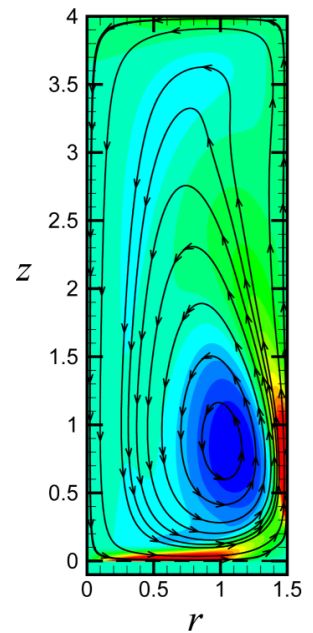

(b)

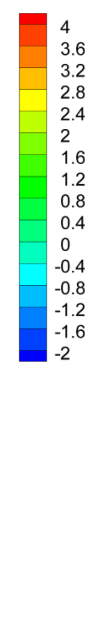

FIG. 4. Contour plots showing azimuthal vorticity and streamlines in different height containers: (a) $H=2$, (b) $H=4$, (c) $H=8$.

coefficient in (12) multiplying the percentage of the incident acoustic intensity that passes into the solid. The maximum temperature within the solid and the maximum and minimum vorticity values are observed to exhibit a high degree of data collapse, whereas the maximum surface temperature at the solid-fluid interface and the maximum velocity magnitude of the acoustic streaming flow have good data collapse for the cases with $R=0.08$ and 0.3 but somewhat weaker collapse for the case with $R=0.6$. A high degree of data collapse for the maximum solid temperature and the minimum negative vorticity makes sense since both acoustic body force (4) and acoustic heat source (7), responsible for generating the solid temperature rise and the negative vorticity, are linear in the acoustic intensity.

It is interesting that while all of the other variables involved in the simulation attain a nearly constant value by about $t=100$, the maximum fluid-solid interface surface temperature $T_{s, \max }$ continues to increase throughout the computation. For this reason, we refer to the flow field that develops as a "quasi-steady state," since not all variables are constant. It is possible that over sufficient time the fluid-solid interface temperature could increase sufficiently so as to cause thermal instability of the flow field, leading the apparent steady-state acoustic streaming flow to transition into a second type of

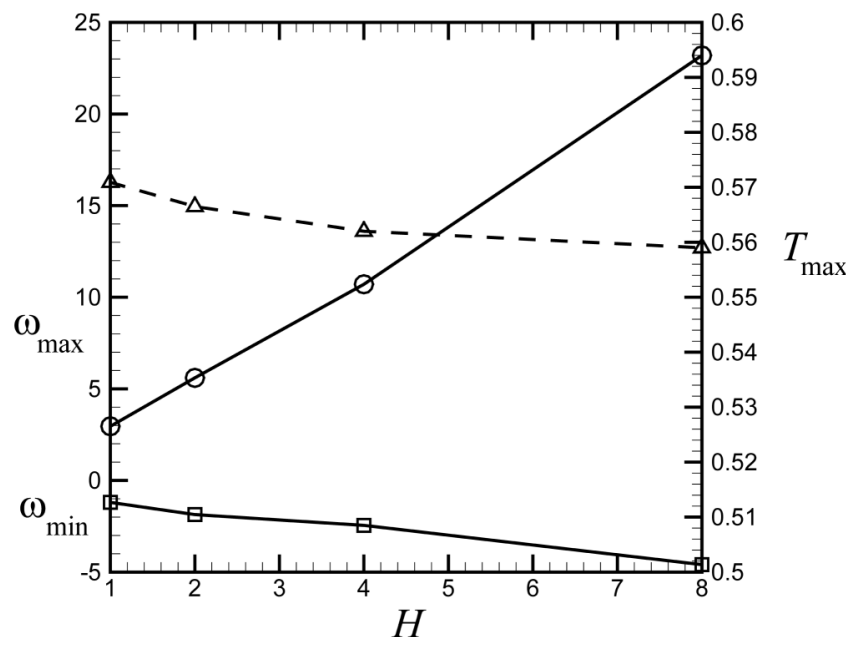

FIG. 5. Plot showing change in maximum (circles) and minimum (squares) azimuthal vorticity values (solid lines, left-hand axis) and maximum substrate temperature (deltas, dashed line, right-hand axis) as functions of height $H$ of the liquid in the cylindrical container. 


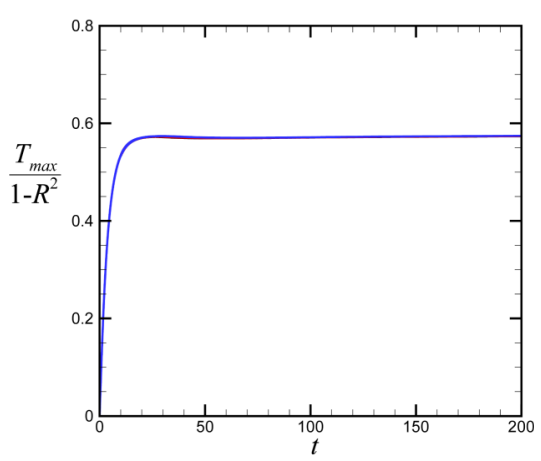

(a)

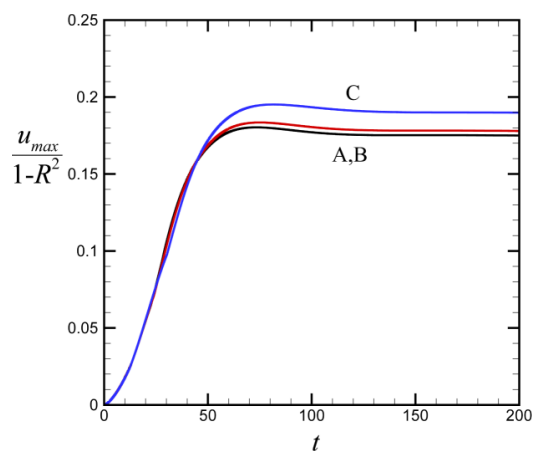

(c)

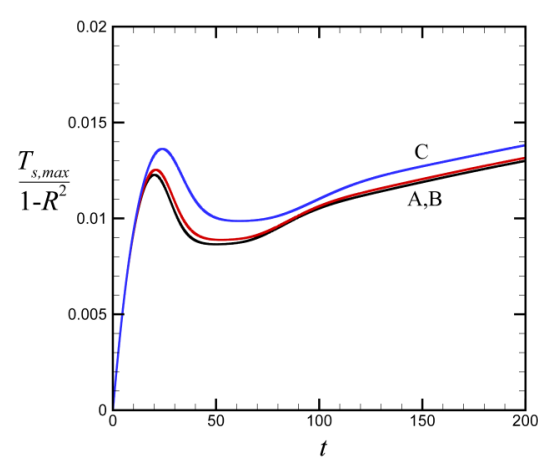

(b)

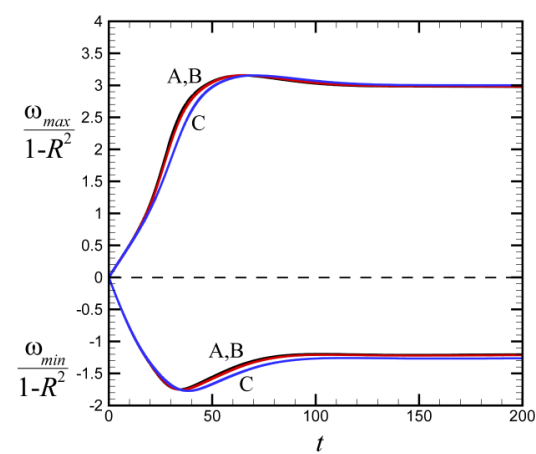

(d)

FIG. 6. Time variation of the (a) maximum temperature within the end-wall solid, (b) end-wall top surface temperature, (c) maximum velocity magnitude, and (d) maximum and minimum vorticity, normalized by the coefficient $\left(1-R^{2}\right)$, for cases with $R=0.08$ (A, black line), 0.3 (B, red line), and 0.6 (C, blue line).

flow field dominated by thermal buoyancy. We did not observe this transition for the time periods and parameter values for which the current computations were conducted, but in a separate experimental study of a similar problem we have observed such a transition after the acoustic streaming state has persisted for sufficient time. ${ }^{23}$

This configuration bears some resemblance to the classic Rayleigh-Bénard problem, involving flow through a two-dimensional channel across which is applied a finite temperature gradient. The Rayleigh number Ra $\equiv \mathrm{Gr} \cdot$ Pr has a critical value of 1707.7 in the inviscid Rayleigh-Bénard problem, whereas the Rayleigh number based on the scaling in Table I for the current problem is 1380. Finite Reynolds number is observed to increase the critical Rayleigh number value. The effect of acoustic streaming on critical Rayleigh number was discussed by Hadid et al..$^{35}$

\section{PARTICLE ROLLING AND REMOVAL FROM THE IMPINGEMENT SURFACE}

Particle transport on the impingement surface is important for applications in which ultrasound is used to clean particles from a surface. ${ }^{16-18}$ Particle transport along the impingement surface is dominated by particle rolling motion driven by the wall shear stress. Collisions of particles can result in some particles being pushed upward off of the impingement surface, allowing them to be entrained into the flow field. ${ }^{50}$ The radial shear stress along the impingement surface is plotted in Figure 7 for the basic flow, as well as for the different container heights examined in Figure 4 and the different reflection coefficients examined in Figure 6. The shape of the shear stress profile is similar in all cases, with zero shear stress at the cylinder axis $(r=0)$ and side wall $(r=L)$, and peak shear stress near the half-way radial location between the axis and the side wall. The shear stress magnitude increases as the container height is increased (approximately linearly in $H$ ) and it decreases as the reflection coefficient is increased (approximately in proportion to the factor $1-R^{2}$ ). For high container heights, we 


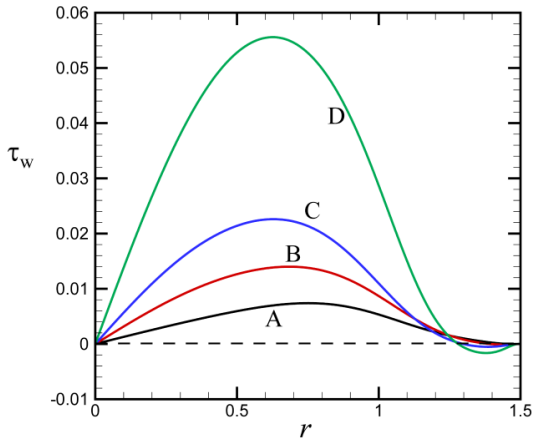

(a)

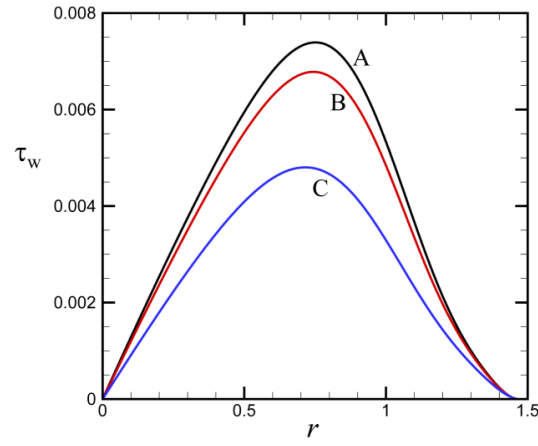

(b)

FIG. 7. Shear stress profile on bottom surface for polyurethane for (a) different container heights $H$ and (b) different reflection coefficients $R$ (b). In (a), we set the parameters as in the basic flow with height $H=1$ (A, black line), 2 (B, red line), 4 (C, blue line), and $8(\mathrm{D}$, green line). In (b), we set the parameters as in the basic flow with reflection coefficient $R=0.08$ (A, black line), 0.3 (B, red line), and 0.6 (C, blue line).

also note the occurrence of a region with negative shear stress near $r=L$, which indicates the presence of a small recirculation region in the corner of the container near $r=L$, as observed in Figure 4.

The particle dynamics on the impingement surface is controlled by the driving torque $M_{d}$ and the resistance torque $M_{r}$, where $M_{d}$ is related to the fluid force on the particle and $M_{r}$ is related to adhesive force between the particle and the impingement surface. The driving torque can be written for a particle with diameter $d$ as

$$
M_{d}=F_{d}(d / 2),
$$

where the fluid drag force is given for cases with small particle Reynolds number by

$$
F_{d}=3 \pi d \mu f(u-v),
$$

and where $\mu$ is the fluid viscosity and $u$ and $v$ are the fluid and particle radial velocities, respectively. The coefficient $f$ represents an adjustment to the Stokes drag law caused by the proximity of the wall, which for a particle resting on the wall is given by King and Leighton ${ }^{51}$ as $f=1.70$. Assuming that the particle diameter is much smaller than the boundary layer thickness at the wall, the fluid velocity can be approximated in terms of the wall shear stress as $u=d \tau_{w} / 2 \mu$.

An expression for the resistance torque under the action of van der Waals adhesion was given by Dominik and Tielens ${ }^{52}$ as

$$
M_{r}=4 F_{C}\left(\frac{a}{a_{0}}\right)^{3 / 2} b_{c r i t},
$$

where $F_{C}=3 \pi \gamma d / 2$ is the critical normal force for particle pull-off given by Johnson-KendallRoberts (JKR) theory, ${ }^{53} \gamma$ is the particle adhesive surface energy, $a$ is the contact region radius with equilibrium value $a_{0}=\left(9 \pi \gamma d^{2} / 4 E\right)^{1 / 3}$, and $E=Y / 2\left(1-\sigma^{2}\right)$ is the effective elastic modulus, which is written in terms of the particle's Young's modulus $Y$ and Poisson ratio $\sigma$. The coefficient $b_{\text {crit }}$ is the critical particle displacement tangent to the surface before onset of rolling. Values of $b_{\text {crit }} / d$ were obtained experimentally by Ding et al. ${ }^{54}$ for polymer microspheres $(7.5 \mu \mathrm{m}$ diameter) and found to lie in the range 0.01-0.03.

Particle rolling does not begin until the driving torque exceeds the resistance torque. Setting $M_{d}=M_{r}$ and assuming that the particle is at equilibrium in the direction normal to the surface, so that $a / a_{0} \cong 1$, gives the critical wall shear stress for onset of particle rolling as

$$
\tau_{\text {crit }}=\frac{8 \gamma}{f d}\left(\frac{b_{\text {crit }}}{d}\right) \cong 0.094 \gamma / d,
$$

where in the latter expression we set $b_{\text {crit }} / d \cong 0.02$. When $\tau_{w}<\tau_{\text {crit }}$, the particles adhere to the surface without rolling. When $\tau_{w}>\tau_{c r i t}$, the particles roll on the surface with a radial velocity $v$, which is obtained by solution of the particle angular momentum equation 


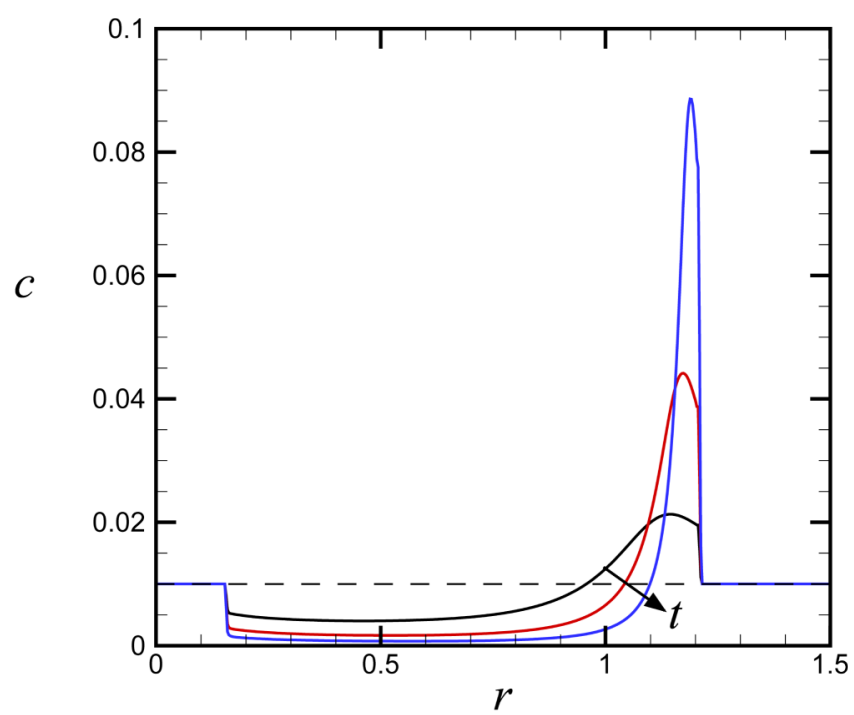

FIG. 8. Evolution of the area-based concentration profile of particles on the impingement surface for the steady-state basic flow with critical shear stress $\tau_{\text {crit }}=0.002$. The dashed line shows the initial concentration field and solid lines are drawn at times $t=100$ (black), 200 (red), and 300 (blue).

$$
I \frac{d \Omega}{d t}=M_{d}-M_{r}
$$

where $I=m d^{2} / 10$ is the particle moment of inertia and $\Omega=2 v / d$ is the particle rotation rate. For small values of the particle Stokes number $\mathrm{St} \equiv \frac{\tau_{p}}{\tau_{f}}=\frac{m U}{3 \pi \mu d L}$, the particle velocity can be approximated by the local equilibrium solution

$$
v_{e q}=\frac{d}{2 \mu}\left(\tau_{w}-\tau_{c r i t}\right)
$$

Here, $m=\pi \rho_{p} d^{3} / 6$ is the particle mass, $\rho_{p}$ is particle density, $U$ and $L$ are characteristic fluid velocity and length scales, and $\tau_{p}$ and $\tau_{f}$ are the particle and fluid time scales.

The area-based concentration $c(r, t)$ is defined as the area on the impingement surface covered by particles divided by the total surface area. In cylindrical polar coordinates, the governing equation for $c$ is given by

$$
\frac{\partial c}{\partial t}+\frac{1}{r} \frac{\partial}{\partial r}(r c v)=0
$$

Numerical simulations for the concentration field were performed by solving (32) using the equilibrium solution (31) for velocity, which assumes small Stokes number and neglects particle collisions. Equation (32) was discretized using a Crank-Nicholson approach with forward differencing in time and centered differencing in space. A plot showing the evolution of the area-based concentration field with time is given in Figure 8 for a case with critical shear stress $\tau_{\text {crit }}=0.002$ and initial concentration $c(r, 0)=0.01$. There is no change in concentration in regions where $\tau_{w}<\tau_{c r i t}$, which are found both near the cylinder axis and near the side wall. In the central region where $\tau_{w}>\tau_{\text {crit }}$, the concentration is observed to decrease with time for small $r$ and increase with time for larger $r$. Over long time, the particles would exhibit an island of "stranded" particles around the cylinder axis, be depleted from the mid-radius region, and exhibit a large spike in concentration just before the point where $\tau_{w}=\tau_{c r i t}$. As the concentration within this spike grows, particles would become increasingly likely to collide with each other, resulting in particles being pushed out into the flow field.

\section{FLUID MIXING}

Ultrasound beams are commonly used to mix fluids within a container. A demonstration of fluid mixing by the acoustic-streaming flow is shown in Figure 9 for a case where the container is initially 


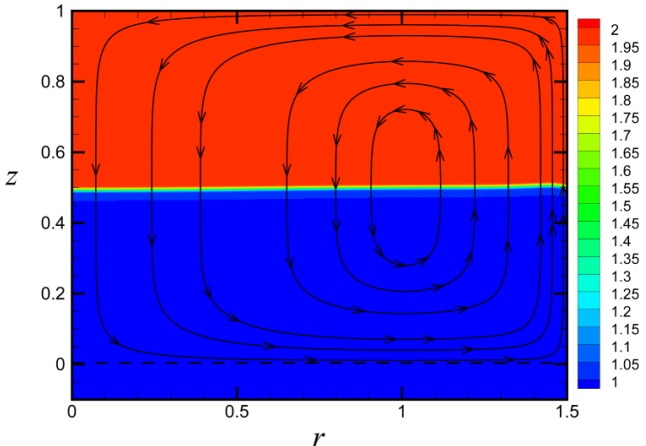

(a)

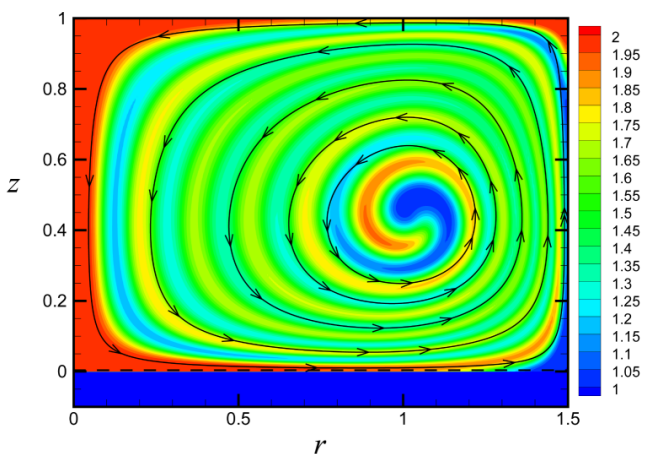

(c)

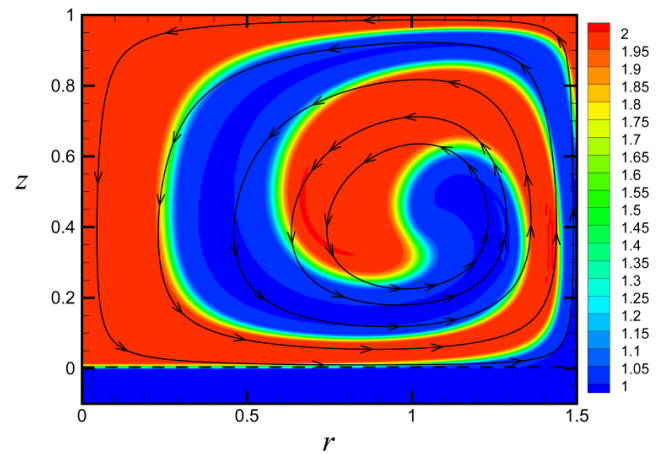

(b)

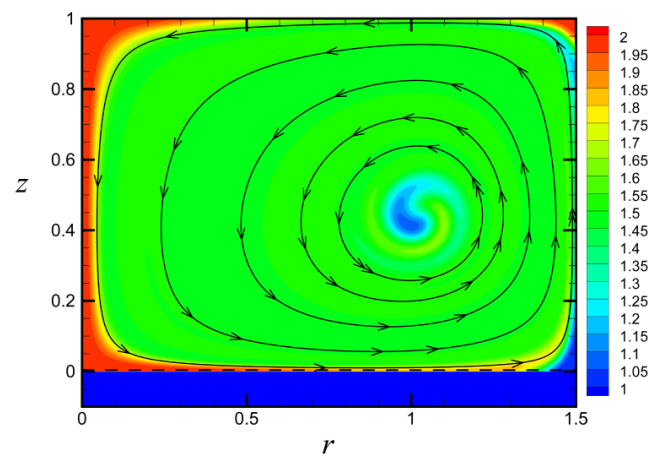

(d)

FIG. 9. Contours of the indicator field $\phi(t, r, z)$ illustrating a mixing process by the acoustic streaming flow following an impulsive start, for the basic flow at times (a) $t=5$, (b) 50, (c) 100 , and (d) 150 .

half filled with one fluid and then filled the rest of the way with a second fluid with the same properties as the first fluid. The two fluids are miscible and diffuse into each other with a Schmidt number $\mathrm{Sc}=v / D=1000$, where $v$ is the fluid kinematic viscosity and $D$ is the mass diffusion coefficient of the fluid. An indicator field $\phi(t, r, z)$ is used to indicate the amount of each fluid present, with $\phi$ initially set equal to unity in the lower fluid and equal to two in the upper fluid. The indicator field is evolved by the dimensionless advection-diffusion equation

$$
\frac{\partial \phi}{\partial t}+u \frac{\partial \phi}{\partial r}+w \frac{\partial \phi}{\partial z}=\frac{1}{\operatorname{ScRe}}\left[\frac{\partial^{2} \phi}{\partial r^{2}}+\frac{1}{r} \frac{\partial \phi}{\partial r}+\frac{\partial^{2} \phi}{\partial z^{2}}\right] .
$$

Two distinct stages of the mixing process are observed in Figure 9. During the first stage (typically called "macro-mixing"), the primary function of the mixing process is to generate increasingly sharp gradients of the $\phi$ field. As the gradients of $\phi$ increase with the formation of thin striations of the two fluids, the rate of diffusion across these striations also increases. During the second stage (typically called "micro-mixing"), the mixing process is dominated by diffusion of the $\phi$ field, resulting at the end of this process in a nearly homogeneous mixture.

Different measures of mixing efficiency have been proposed by a wide range of investigators, and a summary and assessment of the different measures is given by Bothe. ${ }^{55} \mathrm{~A}$ common measure of degree of mixing is given by the root-mean-square $\phi_{r m s}$ of the indicator function, defined by

$$
\phi_{r m s}^{2}=\frac{2}{H L^{2}} \int_{0}^{H} \int_{0}^{L}[\phi(t, r, z)-\bar{\phi}]^{2} r d r d z,
$$

where the mean value is computed from 


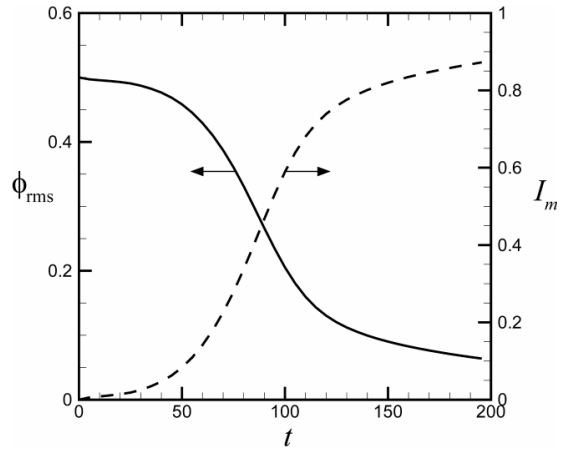

(a)

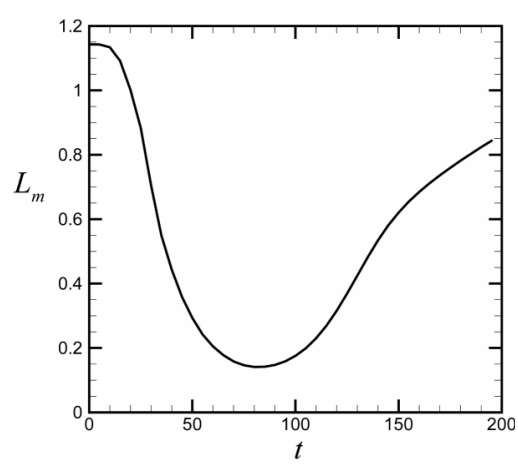

(b)

FIG. 10. Plot showing time variation of (a) the root-mean-square of the indicator field and the mixing measure $I_{m}$ and (b) the mixing length scale for the mixing process shown in Figure 9.

$$
\bar{\phi}=\frac{2}{H L^{2}} \int_{0}^{H} \int_{0}^{L} \phi(t, r, z) r d r d z .
$$

An intensity of mixing $I_{m}$ can then be defined by

$$
I_{m}=1-\sqrt{I_{S}}=1-\frac{\phi_{r m s}}{\phi_{r m s, \max }}
$$

where $I_{S}=\phi_{r m s}^{2} / \phi_{r m s, \max }^{2}$ is Danckwert's intensity of segregation measure ${ }^{56}$ and the maximum variance $\phi_{r m s, \max }^{2}$ for a segregated binary mixture is given by $\phi_{r m s, \text { max }}^{2}=\bar{\phi}(1-\bar{\phi})$. A plot showing time variation of both $\phi_{r m s}$ and $I_{m}$ is given in Figure 10(a).

Since $\phi_{r m s}=\phi_{r m s, \max }$ for any completely segregated system, the above measures are only useful for indicating the amount that one fluid has diffused into the other (micro-mixing), and not the initial stage in which thin striations are formed (macro-mixing). A measure characterizing the macro-mixing stage was suggested by Bothe ${ }^{55}$ called the integral scale of segregation, denoted by $L_{m}$. This measure is given for the current problem by

$$
L_{m}^{-1}=\frac{2}{H L^{2}} \int_{0}^{H} \int_{0}^{L}\|\nabla \phi\| r d r d z .
$$

A plot of $L_{m}$ as a function of time is shown in Figure 10(b). The plots in Figure 10 demonstrate the two stages of mixing quite clearly. During the macro-mixing stage (corresponding to an approximate time interval $0<t<50$ ), the values of $\phi_{r m s}$ and $I_{m}$ are nearly constant, but the mixing length scale $L_{m}$ decreases rapidly to a value of about $20 \%$ of its initial value. During the micro-mixing stage (corresponding to an approximate time interval $50<t<120$ ), the mixing length scale $L_{m}$ remains approximately constant while the value of $I_{m}$ increases from close to zero to about 0.8 . This stage of rapid mixing is then followed by a period of more gradual mixing as the fluids become completely homogeneous, during which the mixing length scale $L_{m}$ increases and $I_{m}$ gradually approaches unity.

One shortcoming of the mixing measures discussed up to now is that they refer to the indicator field $\phi$ and thus are specific to a particular initial configuration of the two fluids. Measures have also been proposed which characterize the potential for a fluid flow to induce mixing which deal only with the flow. Since the formation of thin fluid striations through a stretching process is an essential part of efficient mixing processes, these fluid measures typically characterize the degree of stretching inherent in the fluid flow. The rate of stretching in a fluid flow is controlled by the rate of deformation tensor $\mathbf{D}$, which is the symmetric part of the velocity gradient. The three eigenvalues of $\mathbf{D}$, denoted by $\lambda_{1}, \lambda_{2}$, and $\lambda_{3}$ where $\lambda_{1} \geq \lambda_{2} \geq \lambda_{3}$, are equal to the maximum, inflection, and minimum values of the rate of logarithmic stretching $\dot{\Lambda} / \Lambda$, respectively, where $\Lambda$ is the stretch of a material line segment along one of the principal directions of $\mathbf{D}$ and for an incompressible flow $\lambda_{1}+\lambda_{2}+\lambda_{3}=0$. 


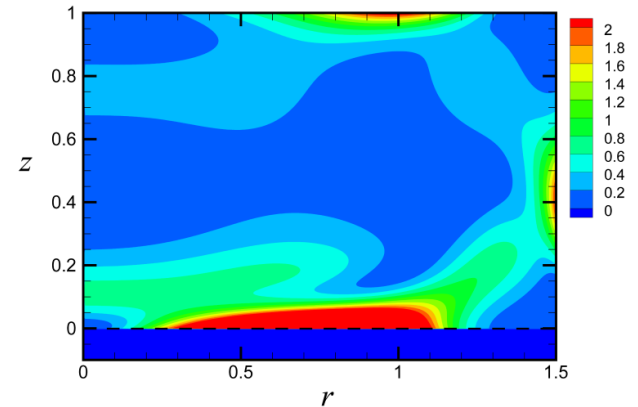

(a)

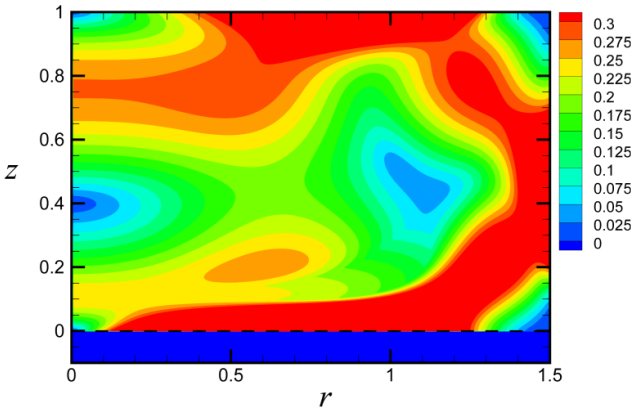

(b)

FIG. 11. Contours of the normalized energy dissipation rate, $\varepsilon / \mu$, and the maximum logarithmic rate of stretching, $\lambda_{1}$, for the case in Figure 9 at steady state.

The potential of a flow for mixing is sometimes assumed to be related to the energy dissipation rate $\varepsilon=2 \mu D_{i j} D_{i j}$, particularly for turbulent flows. The rate of dissipation can be written in terms of the eigenvalues of $\mathbf{D}$ as

$$
\varepsilon / \mu=2 D_{i j} D_{i j}=2\left(\lambda_{1}^{2}+\lambda_{2}^{2}+\lambda_{3}^{2}\right) .
$$

An alternative mixing measure is given by the largest eigenvalue $\lambda_{1}$ of $\mathbf{D}$, which is equal to the maximum value of the rate of logarithmic stretching at a point. Since $\mathbf{D}$ is symmetric, the eigenvalues of $\mathbf{D}$ can be computed very efficiently using the Smith algorithm. ${ }^{57}$

An example showing contour plots for both the rate of dissipation $\varepsilon / \mu$ and the largest eigenvalue $\lambda_{1}$ of $\mathbf{D}$ for the steady-state flow field is given in Figure 11. These plots can be used to identify regions of high and low stretching rate in the fluid flow. The contours in both plots are very similar, as might be expected from relationship (38). The average value of $\lambda_{1}$ over the flow field gives a global measure of the degree of stretching in a fluid flow, which we denote by $S_{\text {ave }}$. A plot of the ratio $S_{\text {ave }} /\left(1-R^{2}\right)$ is given in Figure 12 for cases with reflection coefficients $R$ ranging from 0.08 to 0.6 . This ratio varies only by about $6 \%$ as the reflection coefficient is changed.

\section{PARTICLE DEPOSITION FROM A SUSPENSION}

Particle deposition on the impingement surface under the basic flow field described in Section III was examined using a three-dimensional adhesive discrete element method (DEM). The computation

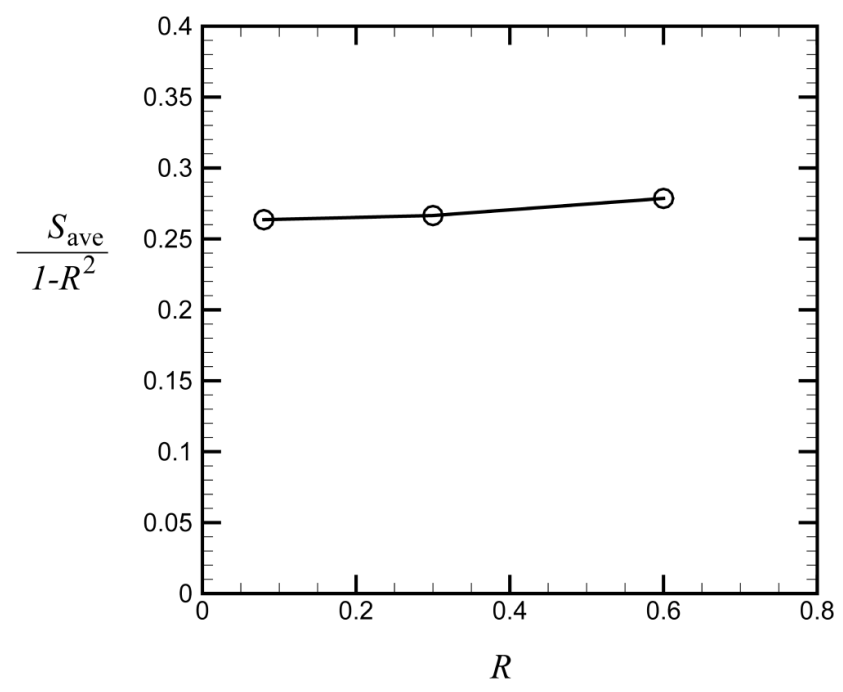

FIG. 12. Plot showing the flow stretching measure $S_{\text {ave }}$, defined as the average value of the largest eigenvalue $\lambda_{1}$ of $\mathbf{D}$ over the flow field, normalized by $1-R^{2}$, as a function of reflection coefficient $R$. 
used the steady-state axisymmetric flow field to specify the fluid velocity field on a three-dimensional grid in a cylindrical polar coordinate system, with 51 grid points in the azimuthal $(\theta)$ direction. The fluid velocity and acoustic intensity on a moving particle were obtained by interpolation from this grid onto the particle location. The particles were transported by a semi-implicit numerical solution of the particle momentum and angular momentum equations, given by

$$
m \frac{d \mathbf{v}}{d t}=\mathbf{F}_{F}+\mathbf{F}_{A}, \quad I \frac{d \boldsymbol{\Omega}}{d t}=\mathbf{M}_{F}+\mathbf{M}_{A},
$$

where $\mathbf{v}$ and $\boldsymbol{\Omega}$ are the particle velocity and rotation rate, $m$ and $I=2 m r_{p}^{2} / 5$ are the mass and moment of inertia of a particle of radius $r_{p}, \mathbf{F}_{F}$ and $\mathbf{M}_{F}$ are the force and torque induced on the particle by the fluid, and $\mathbf{F}_{A}$ and $\mathbf{M}_{A}$ are the force and torque induced on the particle by the sum of adhesion and elastic collision effects. The adhesion and collision forces were obtained using a soft-sphere DEM, with the normal elastic force determined by the well-known JKR theory ${ }^{53}$ and the Dominik and Tielens expression (27) used for the rolling resistance torque. ${ }^{52}$ A normal damping force model of Tsuji et al. ${ }^{58}$ with zero restitution coefficient was used to account for both fluid and viscoelastic damping within the solid. A sliding resistance force and torque and a twisting resistance torque were also used to inhibit sliding and twisting motion, although generally for small particles rolling dominates over sliding motions. A complete account of the adhesive DEM theory was given by Marshall, ${ }^{59}$ to which we refer the reader for details on the governing equations and numerical methods.

The fluid forces are dominated by the drag force $F_{d}$ and the acoustic radiation force $F_{a}$. Since the particle Reynolds number is much smaller than unity and the particle concentration is small, the Stokes drag force expression (27), with $f=1$, can be used. The fluid viscous torque on the particle is given by

$$
\mathbf{M}_{F}=\pi \mu d^{3}\left(\frac{1}{2} \boldsymbol{\omega}-\mathbf{\Omega}\right)
$$

where $\boldsymbol{\omega}$ is the fluid vorticity vector at the particle location.

A detailed study of the acoustic radiation force in a viscous fluid was presented by Doinikov ${ }^{60}$ and more recently by Xie and Vanneste. ${ }^{61}$ For the present computations, we consider neutrally buoyant particles with nominal $20 \mu \mathrm{m}$ diameter immersed in a liquid and subject to ultrasound waves with frequency $f=2.2 \mathrm{~Hz}$ and wavenumber $k=8980 \mathrm{~m}^{-1}$. For this case, the ratio of the particle radius to the acoustic wavelength $\lambda=2 \pi / k$ is $r_{p} / \lambda \cong 0.015<<1$. The viscous penetration length is $\delta=\sqrt{2 v / \omega}$, where $\omega=2 \pi f$ is the circular frequency, so that the ratio of the particle radius to the viscous penetration length is $r_{p} / \delta \cong 900>>1$. For the regime where $r_{p} / \lambda<<1<<r_{p} / \delta$, Doinikov $^{60}$ shows that the magnitude of the leading-order acoustic pressure force on the particle is given by

$$
F_{a}=\frac{6 \pi \rho_{f}|A|^{2}\left(r_{p} k\right)^{3} \delta}{(2+\chi)^{2} r_{p}}(1-\chi)^{2},
$$

where $\chi=\rho_{f} / \rho_{p}$ is the ratio of fluid to particle density and $A$ is the acoustic wave amplitude. The acoustic amplitude is related to the acoustic intensity magnitude $I$ by

$$
|A|^{2}=\frac{2 I}{\rho_{f} c k^{2}}
$$

where $c$ is the speed of sound. For a neutrally buoyant particle, $\chi=1$ and the force in (41) vanishes. For this case, Doinikov ${ }^{60}$ shows that the leading-order expression reverts to the inviscid King equation, ${ }^{62}$ which can be written in terms of the acoustic intensity using (42) as

$$
\mathbf{F}_{a}=\frac{4 \pi}{9} \frac{r_{p}^{2}\left(k r_{p}\right)^{4}}{c} \mathbf{I}
$$

Defining the Stokes number St $=4 \rho_{p} r_{p}^{2} U / 18 \mu b$ and using the characteristic fluid velocity $U$ given by (13), the low Stokes-number approximation $|\mathbf{u}-\mathbf{v}|=O($ St $U)$ for the particle slip velocity ${ }^{63}$ can be used to write the ratio of the acoustic pressure force to the drag force as 


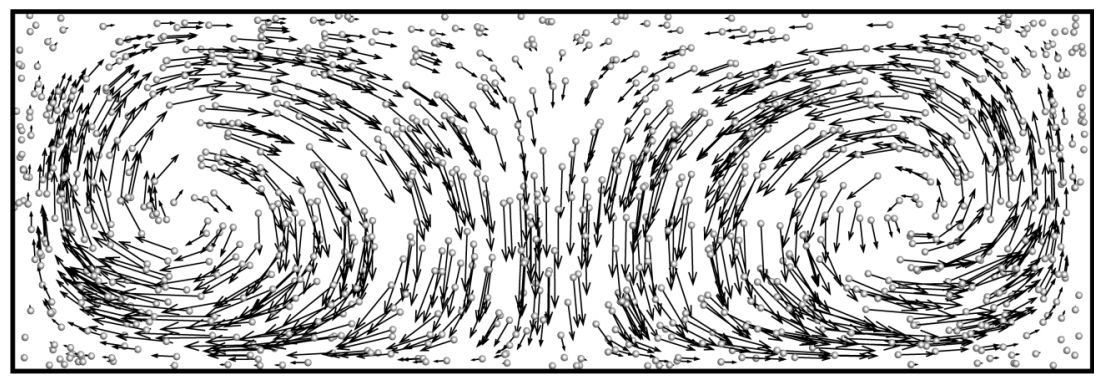

FIG. 13. Plot showing particles and associated particle velocity vectors in the $x-y$ plane, for a slice $-0.05 \leq z \leq 0.05$ of the computational domain. The particles are magnitude by a factor of 5 to make them visible. The transducer is located at the top of the figure and the impingement surface is along the bottom.

$$
\frac{F_{a}}{F_{d}} \sim \frac{2}{27} \frac{r_{p}\left(r_{p} k\right)^{4} I}{\mu c U \mathrm{St}} .
$$

For the case under consideration, the particle size is sufficiently small that the acoustic radiation force has only a small effect on the particle motion. ${ }^{64}$

Simulations were performed with 25675 particles which were initially distributed randomly within the computational domain. A dimensionless adhesion parameter $\Phi$ is used to characterize the magnitude of particle adhesive force relative to the particle inertia, defined by

$$
\Phi=\frac{2 \gamma}{\rho_{p} r_{p} U^{2}},
$$

where $\gamma$ is the particle adhesive surface energy density. Based on typical values of $\gamma$ and the parameters listed in Table I, the adhesion parameter was selected to have a value of order unity for the reported computations, although computations with $\Phi=0.1$ and 10 were also performed.

A side view showing the particles and the particle velocity vectors at time $t=100$ is given in Figure 13. The acoustic transducer is located at the top of the figure and the impingement surface is at the bottom of the figure. Only particles in the slice $-0.05 \leq z \leq 0.05$ are plotted in this figure. The particle velocity vectors follow closely to the associated fluid velocity field due to the small value of the Stokes number. We observe particles with very small velocity vector in the corners of the domain and near the stagnation point as the acoustic streaming jet approaches the impingement surface. The particles touching the impingement surface (i.e., captured particles) are counted as a function of time and plotted in Figure 14(a) for cases with $\Phi=0.1,1$, and 10. The number of captured particles increases in a nearly linear fashion with time throughout the computation, although the overall number

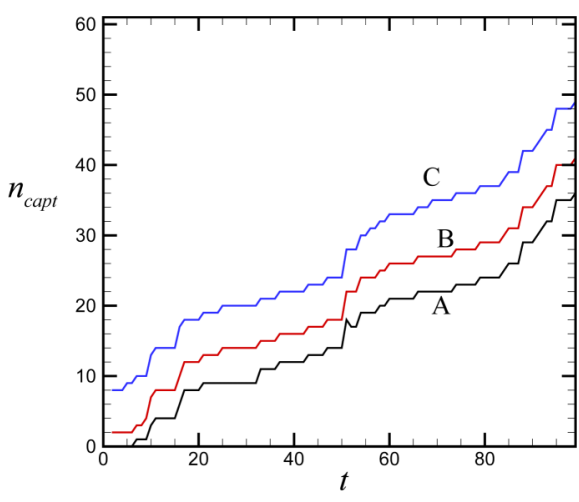

(a)

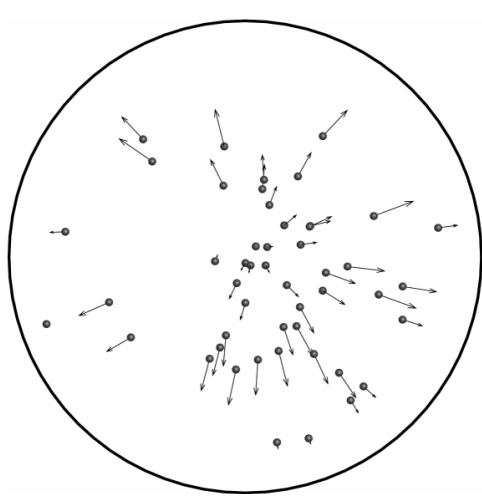

(b)

FIG. 14. (a) Plot of the number of particles captured by the impingement surface as a function of time for cases with $\Phi=0.1$ (A, black line), 1.0 (B, red line), and 10 (C, blue line). (b) Top view of the particles touching the impingement surface at $t=100$, and their associated velocity vectors. 
remains small. A plot showing the motion of the captured particles on the impingement surface is given in Figure 14(b), showing the velocity vectors associated with each particle. By viewing movies of the captured particle motion, it is observed that particles on the impingement surface remain once they have been captured and they slowly roll in the outward radial direction under the action of the fluid flow. Particles close to the central stagnation point and particles close to the lateral wall exhibit very little rolling motion, since in agreement with the results shown in Figure 8, the fluid shear stress in these regions is too small to counteract the adhesive rolling resistance. New particles are gradually added to the set of captured particles during the simulation time.

\section{CONCLUSIONS}

The problem of fluid mixing and particle transport caused by the acoustic streaming flow generated by a Gaussian ultrasound beam oriented along the axis of a closed cylindrical container was examined using numerical simulations. The problem is relevant to a wide range of applications in which acoustic radiation is used to mix liquids in a container, or in which ultrasound is used to clean a surface or to enhance deposition of particles from solution onto a surface. The simulations were performed using an axisymmetric vorticity-streamfunction approach, which was supplemented by temperature computation both within the fluid and within the underlying solid.

The flow field generated by the acoustic streaming motion exhibits a downward-flowing jet that impinges on the end wall of the cylindrical container (called the impingement surface), as well as an upward-flow recirculation along the sides of the container to conserve mass. The overall flow field has the form of a vortex ring in a bounded cylindrical regime. Increase in the container height causes the fluid velocity to increase, since a longer expanse of the flow is subject to the acoustic body force, but the qualitative nature of the flow is unchanged. A series of cases were examined with different values of the impingement surface reflection coefficient $R$, and it is found that the fluid vorticity and velocity fields and the solid temperature scale reasonably well with the intensity transmission factor $1-R^{2}$. The fluid shear stress on the impingement surface was examined and shown to transport particles radially outward, except for within a region near the cylinder symmetry axis and near the cylinder wall, within which regions the shear stress is too weak to overcome the adhesive resistance to particle rolling. It was shown that this flow can rapidly mix the fluid regions within the cylindrical container, but that deposition of particles onto the bottom surface occurs at a much slower pace when the particles are small (e.g., $20 \mu \mathrm{m}$ diameter). When the particles are sufficiently large for the acoustic radiation force to be of the same order of magnitude as the fluid drag force, the particle settling rate would be much more rapid.

\section{ACKNOWLEDGMENTS}

This work has been supported by NASA under cooperative Agreement No. NNX13AD40A.

${ }^{1}$ C. M. Sayers and R. L. Grenfell, "Ultrasonic propagation through hydrating cements," Ultrasonics 31(3), 147-153 (1993).

${ }^{2}$ R. D’Angelo, T. J. Plona, L. M. Schwartz, and P. Coveney, "Ultrasonic measurements on hydrating cement slurries,” Adv. Cem. Based Mater. 2, 8-14 (1995).

${ }^{3}$ L. A. Kuznetsova and W. T. Coakley, "Applications of ultrasound streaming and radiation force in biosensors," Biosens. Bioelectron. 22, 1567-1577 (2007).

${ }^{4}$ G. G. Yaralioglu, I. O. Wygant, T. C. Marentis, and B. T. Khuri-Yakub, "Ultrasonic mixing in microfluidic channels using integrated transducers," Anal. Chem. 76, 3694-3698 (2004).

${ }^{5}$ M. Rahimi, B. Aghel, B. Hatamifar, M. Akbari, and A. Alsairafi, "CFD modeling of mixing intensification assisted with ultrasound wave in a T-type microreactor," Chem. Eng. Process. 86, 36-46 (2014).

${ }^{6}$ N. F. C. Pa, N. L. Chin, Y. A. Yusof, and N. A. Aziz, "Power ultrasound assisted mixing effects on bread physical properties," Agric. Agric. Sci. Procedia 2, 60-66 (2014).

${ }^{7}$ T. Y. Hwang, H. Kim, H. Kim, and J. W. Lee, "Development of branched polycarbonate by an ultrasound-assisted melt mixing process with multifunctional agents," in Proceedings of the XV International Congress on Rheology, Monterey, California, August 3-8 (2008), edited by Albert et al. (AIP, New York, 2008).

${ }^{8}$ B. Verhaagen, C. Boutsioukis, L. W. M. van der Sluis, and M. Versluis, "Acoustic streaming induced by an ultrasonically oscillating endodontic file,” J. Acoust. Soc. Am. 135(4), 1717-1730 (2014).

${ }^{9}$ R. S. Davidson, J. D. Safdar, J. D. Spencer, and B. Robinson, “Applications of ultrasound to organic chemistry,” Ultrasonics 25, 35-39 (1987). 
${ }^{10}$ H. Monnier, A. M. Wilhelm, and H. Delmas, "Effects of ultrasound on micromixing in flow cell," Chem. Eng. Sci. 55, 4009-4020 (2000).

${ }^{11}$ Z. Yang, S. Matsumoto, H. Goto, M. Matsumoto, and R. Maeda, "Ultrasonic micromixer for microfluidic systems," Sens. Actuators, A 93, 266-272 (2001).

12 T. H. Kuehn, T. B. Kittelson, Y. Wu, and R. Gouk, "Particle removal from semiconductor wafers by megasonic cleaning," J. Aerosol Sci. 47, S427-S428 (1996).

${ }^{13}$ W. Kim, T. H. Kim, J. Choi, and H. Y. Kim, "Mechanism of particle removal by megasonic waves," Appl. Phys. Lett. 94, 081908 (2009).

${ }^{14}$ F. Zhang, A. A. Busnaina, M. A. Fury, and S. Q. Wang, "The removal of deformed submicron particles from silicon wafers by spin rinse and megasonics," J. Electron. Mater. 29(2), 199-204 (2000).

${ }^{15} \mathrm{H}$. O. Lamminen, H. W. Walker, and L. K. Weavers, "Mechanisms and factors influences the ultrasonic cleaning of particle-fouled ceramic membranes," J. Membr. Sci. 237, 213-223 (2004).

${ }^{16}$ M. Keswani, S. Raghavan, P. Deymier, and S. Verhaverbeke, "Megasonic cleaning of wafers in electrolytic solutions: Possible role of electro-acoustic and cavitation effects," Microelectron. Eng. 86, 132-139 (2009).

${ }^{17} \mathrm{D}$. Chen and J. R. Wu, "Dislodgement and removal of dust-particles from a surface by a technique combining acoustic standing wave and airflow," J. Acoust. Soc. Am. 127, 45-50 (2010).

${ }^{18}$ A. Fuhrmann, J. S. Marshall, and J. R. Wu, "Effect of acoustic levitation force on aerodynamic particle removal from a surface," Appl. Acoust. 74, 535-543 (2013).

19 J. Lei, P. Glynne-Jones, and M. Hill, "Acoustic streaming in the transducer plane in ultrasonic particle manipulation devices," Lab Chip 13(11), 2133-2143 (2013).

${ }^{20}$ C. Devendran, I. Gralinski, and A. Neild, "Separation of particles using acoustic streaming and radiation forces in an open microfluidic channel," Microfluid. Nanofluid. 17, 879-890 (2014).

${ }^{21}$ S.-L. Huang, "Liposomes in ultrasonic drug and gene delivery," Adv. Drug Delivery Rev. 60(10), 1167-1176 (2008).

${ }^{22}$ A. Schroeder, J. Kost, and Y. Barenholz, "Ultrasound, liposomes, and drug delivery: Principles for using ultrasound to control the release of drugs from liposomes," Chem. Phys. Lipids 162, 1-16 (2009).

${ }^{23}$ D. Ma, A. M. Green, G. G. Willsey, J. S. Marshall, M. J. Wargo, and J. R. Wu, "Effects of acoustic streaming from moderateintensity pulsed ultrasound for enhancing biofilm mitigation effectiveness of drug-loaded liposomes," J. Acoust. Soc. Am. 138(2), 1043-1051 (2015)

${ }^{24}$ M. Wiklund, J. Toivonen, M. Tirri, P. Hänninen, and H. M. Hertz, "Ultrasonic enrichment of microspheres for ultrasensitive biomedical analysis in confocal laser-scanning fluorescence detection," J. Appl. Phys. 96(2), 1242-1248 (2004).

${ }^{25}$ P. Agrawal, P. S. Gandhi, and A. Neild, "Microparticle response to two-dimensional streaming flows in rectangular chambers undergoing low-frequency horizontal vibrations," Phys. Rev. Appl. 2, 064008 (2014).

${ }^{26}$ J. S. Marshall, "Particle clustering in periodically-forced straining flows," J. Fluid Mech. 624, 69-100 (2009).

${ }^{27}$ M. K. Aktas and B. Farouk, "Numerical simulation of acoustic streaming generated by finite-amplitude resonant oscillations in an enclosure," J. Acoust. Soc. Am. 116(5), 2822-2831 (2004).

${ }^{28}$ M. K. Aktas and T. Ozgumus, "The effects of acoustic streaming on thermal convection in an enclosure with differentially heated horizontal walls," Int. J. Heat Mass Transfer 53, 5289-5297 (2010).

${ }^{29}$ V. Daru, D. Baltean-Carlès, C. Weisman, P. Debesse, and G. Gandikota, "Two-dimensional numerical simulations of nonlinear acoustic streaming in standing waves," Wave Motion 50, 955-963 (2013).

${ }^{30}$ M. Wiklund, R. Green, and M. Ohlin, "Applications of acoustic streaming in microfluidic devices," Lab Chip 12, 2438-2451 (2012).

${ }^{31}$ C. Suri, K. Takenaka, H. Yanagida, Y. Kojima, and K. Koyama, "Chaotic mixing generated by acoustic streaming," Ultrasonics 40, 393-396 (2002)

${ }^{32}$ H. Mitome, T. Kozuka, and T. Tuziuti, "Measurement of the establishment process of acoustic streaming using laser Doppler velocimetry," Ultrasonics 34, 527-530 (1996).

${ }^{33}$ W. Dridi, D. Henry, and H. B. Hadid, "Influence of acoustic streaming on the stability of a laterally heated three-dimensional cavity," Phys. Rev. E 77, 046311 (2008).

${ }^{34}$ W. Dridi, D. Henry, and H. B. Hadid, "Stability of buoyant convection in a layer submitted to acoustic streaming," Phys. Rev. E 81, 056309 (2010).

${ }^{35}$ H. B. Hadid, W. Dridi, V. Botton, B. Moudjed, and D. Henry, "Instabilities in the Rayleigh-Bénard-Eckart problem," Phys. Rev. E 86, 016312 (2012).

${ }^{36}$ P. B. Muller and H. Bruus, "Numerical study of thermoviscous effects in ultrasound-induced acoustic streaming in microchannels," Phys. Rev. E 90, 043016 (2014).

${ }^{37}$ W. L. Nyborg, "Acoustic streaming," in Physical Acoustics, IIB, edited by W. P. Mason (Academic Press, New York, 1965), pp. 265-331.

${ }^{38}$ S. I. Aanonsen, T. Barkev, J. N. Tjotta, and S. Tjotta, "Distortion and harmonic generation in the nearfield of a finite amplitude sound beam," J. Acoust. Soc. Am. 75, 749-768 (1984).

${ }^{39}$ L. Filipczynski and J. Etienne, "Theoretical study and experiments on spherical focusing transducers with Gaussian surface velocity distribution," Acustica 28, 121-128 (1973).

40 J. Lighthill, “Acoustic streaming," J. Sound Vib. 61(3), 391-418 (1978).

${ }^{41}$ J. Wu and G. Du, "Acoustic streaming generated by a focused Gaussian beam and finite amplitude tonebursts," Ultrasound Med. Biol. 19(2), 167-176 (1993).

42 B. Moudjed, V. Botton, D. Henry, H. Ben Hadid, and J.-P. Garandet, "Scaling and dimensional analysis of acoustic streaming jets," Phys. Fluids 26, 093602 (2014).

43 J. Wu and W. L. Nyborg, "Temperature rise generated by a focused Gaussian beam in a two-layer medium," Ultrasound Med. Biol. 18(3), 293-302 (1992).

${ }^{44}$ B. Moudjed, V. Botton, D. Henry, S. Millet, J. P. Garandet, and H. B. Hadid, "Near-field acoustic streaming jet," Phys. Rev E 91, 033011 (2015) 
45 A. N. Norris, "Back reflection of ultrasonic waves from a liquid-solid interface," J. Acoust. Soc. Am. 73(2), 427-434 (1983).

${ }^{46}$ G. Du and M. A. Breazeale, "Theoretical description of a focused Gaussian ultrasonic beam in a nonlinear medium," J. Acoust. Soc. Am. 81(1), 51-57 (1987).

${ }^{47}$ F. Duck, Physical Properties of Tissue: A Comprehensive Reference Book (Academic Press, London, 1990).

${ }^{48}$ R. Peyret and T. D. Taylor, Computational Methods for Fluid Flow (Springer-Verlag, New York, 1983), pp. 184-185.

${ }^{49}$ P. H. Mott and R. C. Roland, "Acoustic and dynamic mechanical properties of a polyurethane rubber," J. Acoust. Soc. Am. 111(4), 1782-1790 (2002)

${ }^{50}$ A. B. Maynard and J. S. Marshall, "Particle removal from a surface by a bounded vortex flow," Int. J. Heat Fluid Flow 32(5), 901-914 (2011).

${ }^{51}$ M. R. King and D. T. Leighton, "Measurement of the inertial lift on a moving sphere on contact with a plane wall in a shear flow," Phys. Fluids 9(5), 1248-1255 (1997).

${ }^{52}$ C. Dominik and A. G. G. M. Tielens, "Resistance to rolling in the adhesive contact of two elastic spheres," Philos. Mag. A 92(3), 783-803 (1995).

${ }^{53}$ K. L. Johnson, K. Kendall, and A. D. Roberts, "Surface energy and the contact of elastic solids," Proc. R. Soc. London, Ser A 324, 301-313 (1971).

${ }^{54}$ W. Ding, H. Zhang, and C. Cetinkaya, "Rolling resistance moment-based adhesion characterization of microspheres," J. Adhes. 84, 996-1006 (2008).

${ }^{55}$ D. Bothe, "Evaluating the quality of a mixture: Degree of homogeneity and scale of segregation," in Micro and Macro Mixing: Analysis, Simulation and Numerical Calculation, edited by H. Bockhorn, D. Mewes, W. Peukert, and H.-J. Warnecke (Springer, Berlin, 2010), pp. 16-35.

${ }^{56}$ P. V. Danckwerts, "The definition and measurement of some characteristics of mixtures," Appl. Sci. Res. A 3, 279-296 (1952).

57 O. K. Smith, "Eigenvalues of a symmetric $3 \times 3$ matrix," Commun. ACM 4(4), 168 (1961).

${ }^{58}$ Y. Tsuji, T. Tanaka, and T. Ishida, "Lagrangian numerical simulation of plug flow of cohesionless particles in a horizontal pipe," Powder Technol. 71, 239-250 (1992).

59 J. S. Marshall, "Discrete-element modeling of particulate aerosol flows,” J. Comput. Phys. 228, 1541-1561 (2009).

${ }^{60}$ A. A. Doinikov, "Acoustic radiation pressure on a rigid sphere in a viscous fluid," Proc. R. Soc. London, Ser. A 447(1931), 447-466 (1994).

${ }^{61}$ J.-H. Xie and J. Vanneste, "Dynamics of a spherical particle in an acoustic field: A multiscale approach,” Phys. Fluids 26, 102001 (2014)

${ }^{62}$ L. V. King, "On the acoustic radiation pressure on spheres," Proc. R. Soc. London, Ser. A 147(861), 212-240 (1934).

${ }^{63}$ J. S. Marshall and S. Li, Adhesive Particle Flow: A Discrete Element Approach (Cambridge University Press, New York, 2014).

${ }^{64}$ R. Barnkob, P. Augustsson, T. Laurell, and H. Bruus, "Acoustic radiation- and streaming-induced microparticle velocities determined by microparticle image velocimetry in an ultrasound plane," Phys. Rev. E 86, 056307 (2012). 